\title{
Performance Analysis of Buffered Banyan ATM Switch Architectures
}

\author{
D. Kouvatsos, J. Wilkinson,
} Computer Systems Modelling Group, Dept. of Computing, University of Bradford, BRADFORD BD7 1DP, U.K.

Tel: 01274383941 Fax: 01274383920

Email: D.D.Kouvatsos, J.Wilkinson@comp.brad.ac.uk,

\section{P. Harrison, M. Bhabuta}

Dept. of Computing, Imperial College of Science, Technology \& Medicine, 180 Queens Gate, LONDON SW7 2BZ, U.K.

Tel: 01715948363 Fax: 01715818024

Email:pgh,mb3@doc.ic.ac.uk

\begin{abstract}
The principle of Maximum Entropy (ME) and the notion of system decomposition are combined towards the creation of an iterative cost-effective approximation algorithm for the performance analysis of packet-switched buffered Banyan Multistage Interconnection Network (MIN) based Asynchronous Transfer Mode (ATM) switch architectures with arbitrary buffer sizes, multiple input/output ports and Repetitive Service (RS) internal blocking.

Traffic entering and flowing in the MIN is assumed to be bursty and it is modelled by a Compound Poisson Process (CPP) with geometrically distributed bulk sizes and Generalised Exponential (GE) interarrival times. The GE distribution is also adopted to represent the random nature of the effective service times of packets due to the combined effects of traffic burstiness and RS blocking.

Entropy maximisation implies decomposition of the Banyan network into individual building block queues of switching elements, represented by shared buffer cross bars, under revised GE-type interarrival and service times. Each building block queue is analysed in isolation by applying ME techniques and classical queueing theory, subject to marginal mean value constraints, in order to obtain a product form solution for the joint queue length distribution and typical performance metrics of the network.

Numerical results are included to validate the credibility of the ME approximation against simulation, define experimental performance bounds and perform a buffer capacity optimisation across the entire network.
\end{abstract}

\footnotetext{
- Supported by the Engineering and Physical Sciences Research Council (EPSRC), UK, under grant GR/K/67809.
} 


\begin{abstract}
Keywords
Multistage Iterconnection Network (MIN), Banyan network, Queueing Network Model (QNM), Repetitive-Service (RS) blocking mechanism, Maximum Entropy (ME) Principle, Compound Poisson Process (CPP), Generalised Exponential (GE) distribution, Asynchronous Transfer Mode(ATM) switch architectures.
\end{abstract}

\title{
1 INTRODUCTION
}

During the past decade, a considerable amount of effort has been made towards the design and development of Asynchronous Transfer Mode (ATM) switch architectures, which are widely considered as the preferred packet-oriented solution of a new generation of high speed communication systems, both for broadband public information highways and for local and wide area private networks (e.g., Tobagi [25]).

Amongst the many types of ATM switch architectures, of particular interest are the so called space division switches which are primarily based on Multistage Interconnection Networks (MINs) (e.g., [1,2,19]). Such switches are composed of smaller switching elements represented by shared-buffer crossbars. Main features of a MIN include non-centralised switching control and multiple concurrent paths in tandem from input ports to output ports.

MINs are also widely employed in parallel processing systems as a means for processor memory (and interprocessor) communication. The nature of traffic in ATM switches, however, is quite different from that observed in typical parallel machines in the sense that, regarding the latter, there is basically only one type of service, namely, high speed data (not considering "probe" and "acknowledgment" signals observed in inter-stage transmissions), whereas for the former, there exists a greater variety of integrated services including voice, low and high speed data, teleconferencing, TV distribution and video on demand, all of which share the same communication medium with different cell loss and delay requirements.

The integration of such ATM services implies considerable variability in terms of transmission speed and holding times. Moreover, the flow of cells through one switching element may be momentarily blocked (halted) if the downstream switching element has reached its buffer capacity. Thus, credible analytical tools are essential for the cost-effective performance modelling prediction of such complex ATM switches.

An increasing number of earlier papers concerning with the performance modelling and analysis of MINs have appeared in the literature (e.g., [4-6, 8, 20, 24, 26]) and such trend is likely to continue towards the design and development of more appropriate ATM space division architectures. In this context, analytic performance models of shared buffer ATM switch architectures, based on both continuous-time and discrete-time queueing models, have received particular attention. Pinto and Harrison $[4,5]$ proposed approximate algorithms for the analysis continuous-time asynchronous buffered Banyan networks with $2 \times 2$ switching elements using Exponential interarrival times and 2-phase Coxian $\left(C_{2}\right)$ and Generalised Exponential (GE) service time distributions, respectively, with Blocking After Service (BAS) (i.e., service is suspended at the output port for a cell which attempts to enter a destination switching element with a full buffer). Hong et al [6] and Yamashita et al [26] described approximate algorithms for the performance evaluation of discrete-time and continuous-time queueing models of shared buffer ATM switches under both Interrupted Bernoulli and Interrupted Poisson arrival processes, respectively. In terms of computational implementation, 
these works tackle the problem by either solving global balance equations numerically $[4,5]$, or by decomposing the switch into several subsystems, each of which being analysed numerically in isolation $[6,26]$. However, as the number of input (or output) ports increases, so does the size of the system's state space, and therefore, further approximations are required in order to achieve, if at all possible, tractable solutions. Thus, there is a great need to apply alternative methodologies leading to both accurate and cost-effective approximations for the performance modelling and evaluation of MIN-based shared buffer ATM switches.

The principle of Maximum Entropy (ME), a probability inference method (c.f., Jaynes [7], Shore and Johnson [22]), has been used successfully, in conjunction with queueing theoretic mean value constraints, for the approximate analysis of both continuous time and discrete time arbitrary Queueing Network Models (QNMs) with single general queues of finite or infinite capacity (e.g., [10-17]). In particular, the principle has been utilised in the study of general multibuffered and shared buffer queues and closed form expressions in both continuous-time and discrete-time domains have been obtained for Queue Length Distributions (QLD), Cell Loss Probabilities (CLP) and mean delays [14, 15]. More recently, a new product from approximation has been established by Kouvatsos and Wilkinson [17], towards the costeffective performance analysis of arbitrary open discrete-time QNMs of shared buffer queues with cell loss. In the afor mentioned studies the arrival process at each queue has been assumed to be highly variable and was modelled by Compound Poisson (CPP) or Bernoulli (CBP) processes, both with geometrically distributed bulk sizes. In this context, the burstiness of the arrival process is characterised by the squared coefficient of variation (SCV) of the interarrival times or, equivalently, the average size of the incoming bulk. The CPP and CBP arrival processes imply GE and Generalised Geometric (GGeo) interarrival-time distributions, respectively, whose pseudo-memoryless properties facilitate the analysis of complex queues and networks (e.g., $[11,13,16])$. The choice of GE and GGeo distributions has been further motivated by the fact that measurements of actual traffic or service times are generally limited and so only few parameters can be computed reliably. Typically, only the mean and variance can be relied upon. In this case, the choice of distributions which imply least bias (c.f., [7]) (i.e., introduction of arbitrary and, therefore, false assumptions) is that of a GE or GGeo distribution within a continuous-time or a discrete time context, respectively.

In this paper queueing network modelling and entropy maximisation are employed towards the performance analysis of Banyan MINs with GE-type external traffic pattern and stage-tostage transmission times, arbitrary switching element sizes $(R \times R, R \geq 2)$ and buffer capacities, K, under Repetitive-Service (RS) (or communication) internal blocking. Such MINs provide full connectivity between a set of input sources and a set of destination nodes. In a Broadband Integrated Services Digital Network (B-ISDN) environment, Banyan MINs can support several different types of traffic concurrently (e.g., data, voice, video). Consequently, traffic models must be able to capture various flow characteristics such as burstiness (e.g. video traffic which has to be batched). In this context, the GE distribution is adopted to represent (in an appropriate fashion) the random nature of the interarrival times and effective service times of packets in the MIN due to the combined inflence of traffic burstiness and RS blocking. Note that in tandem configurations RS blocking occurs when a cell upon service completion at queue $\kappa$ attempts to join a downstream queue $\ell$ whose buffer capacity is full. Consequently, the cell is rejected by queue $\ell$ and immediately receives another service at queue $\kappa$. This is 
repeated until the cell completes service at queue $\kappa$ at the moment where the destination queue $\ell$ is not full.

Entropy maximisation implies a decomposition of the Banyan network into individual multiple input GE-type shared buffer queues of switching elements with revised (effective) interarrival and transmission times. These queues are solved in isolation and together with GEtype formulae for the first two moments of the cell interdeparture and aggregated arrival processes at each output port queue, play the role of cost effective building blocks towards the performance analysis of the entire network.

The ME formalism is introduced in Section 2. The GE-type distribution is described in Section 3. An ME QLD of a multiple input shared buffer building block queue is outlined in Section 4. An ME product form approximation for a arbitrary QNM of a buffered Banyan MIN together with a description of the traffic flow through the switching elements are presented in Section 5. ME Analysis of three types of switching elements, acting as building blocks, together with appropriate GE flow formulae are presented in Section 6. Section 7 presents the ME approximation algorithm for the performance analysis of arbitrary size Banyan networks. Numerical results and concluding comments follow in Sections 8 and 9, respectively.

\section{MAXIMUM ENTROPY FORMALISM}

Consider a system $Q$ which has a set $S$ of possible discrete states $\left\{S_{0}, S_{1}, S_{2}, \ldots\right\}$ which may be finite or countably infinite and state $S_{n}, n=0,1,2, \ldots$ may be specified arbitrarily. Suppose that the available information about $Q$ places a number of constraints on $p\left(S_{n}\right)$, the probability distribution that the system $Q$ is in state $S_{n}$. Without loss of generality, it is assumed that these constraints take the form of mean values of suitable functions $\left\{f_{1}\left(S_{n}\right), f_{2}\left(S_{n}\right), \ldots, f_{m}\left(S_{n}\right)\right\}$, where $m$ is less than the number of possible states. The principle of maximum entropy [7] states that, of all distributions which satisfy the constraints, the minimally biased distribution is the one which maximises the system's entropy function

$$
\mathrm{H}(p)=-\sum_{S_{n} \in S} p\left(S_{n}\right) \ln p\left(S_{n}\right),
$$

subject to the constraints

$$
\begin{aligned}
& \sum_{S_{n} \in S} p\left(S_{n}\right)=1, \\
& \sum_{S_{n} \in S} \mathrm{f}_{k}\left(S_{n}\right) p\left(S_{n}\right)=\left\langle\mathrm{f}_{k}\right\rangle, \quad k=1,2, \ldots, m,
\end{aligned}
$$

where $\left\{\left\langle\mathrm{f}_{k}\right\rangle: k=1,2, \ldots, m\right\}$ are the prescribed mean values defined on the set of $\mathrm{m}$ functions $\left\{\mathrm{f}_{k}\left(S_{n}\right): k=1,2, \ldots, m\right\}$, where $\mathrm{m}$ is less thatn the number of states in $\mathrm{S}$. The maximisation of 
(2.1), subject to the constraints (2.2) and (2.3), can be carried out using Lagrange's method of undermined multipliers and leads to the solution

$$
p\left(S_{n}\right)=\frac{1}{Z} \exp \left\{-\sum_{k=1}^{m} \beta_{k} \mathrm{f}_{k}\left(S_{n}\right)\right\},
$$

where $\left\{\beta_{k}: k=1,2, \ldots m\right\}$, are the Lagrangian multipliers determined from the set of constraints (2.3) and $Z$, known in statistical physics as the "partition function", is given by

$$
Z=\exp \left(\beta_{0}\right)=\sum_{S_{n} \in S} \exp \left\{-\sum_{k=1}^{m} \beta_{k} \mathrm{f}_{k}\left(S_{n}\right)\right\}
$$

where $\left\{\beta_{0}\right\}$ is the Lagrangian multiplier determined by the normalisation constraint (2.2).

Jaynes [7] has shown that, if the prior information includes all constraints actually operative during a random experiment, the distribution predicted by the maximum entropy can be realised in overwhelmingly more ways than by any other distribution. The principle of maximum entropy has also been shown by Shore and Johnson [22] to provide a "uniquely correct self-consistent method of inference" for estimating probability distributions based on the available information.

Maximum entropy formalism can be applied in the performance analysis of queueing systems because expected values of various distributions of interest are usually known in terms of moments of the interarrival and service time distributions. A review of entropy maximisation for approximate analysis of queueing systems and networks can be seen in Kouvatsos [16].

\section{THE GE DISTRIBUTION}

The GE distribution is of the form

$$
F(t)=P(X \leq t)=1-\tau e^{-\sigma t}, t \geq 0
$$

where

$$
\tau=2 /\left(C^{2}+1\right), \quad \sigma=\tau v,
$$

$\mathrm{X}$ is a mixed-time random variable (rv) of the interevent-time, while $1 / \mathrm{v}$ is the mean and $\mathrm{C}^{2}$ is the SCV of rv X (see Figure 1). 


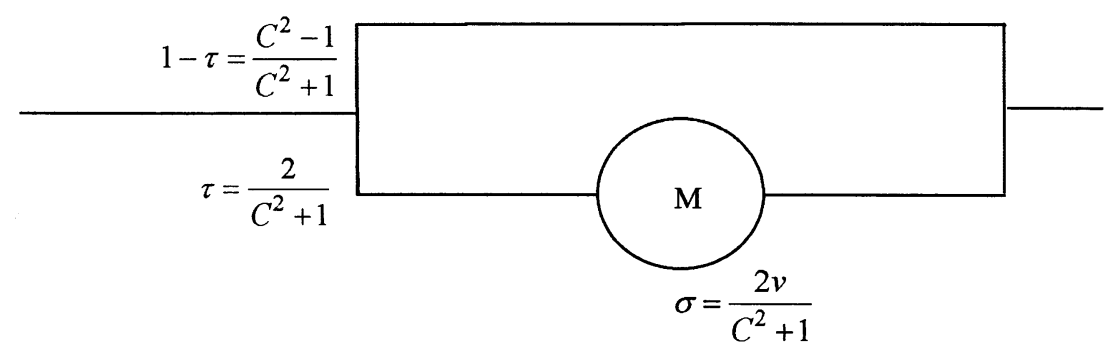

Figure 1 The $G E\left(v, C^{2}\right)$ distribution with parameters $\tau$ and $\sigma$.

For $C^{2} \geq 1$, the GE model is a mixed-time probability distribution and it can be interpreted as either

1. an extremal case of the family of two-phase exponential (M) distributions (e.g., Hyperexponential-2 $\left(\mathrm{H}_{2}\right)$ ) having the same $\mathrm{v}$ and $\mathrm{C}^{2}$, where one of the two phases has zero service time, or

2. a bulk type distribution with an underlying counting process equivalent to a Compound Poisson Process (CPP) with parameter $2 v / C^{2}+1$ and geometrically distributed bulk sizes with mean $=\left(C^{2}+1\right) / 2$ and $\mathrm{SCV}=\left(C^{2}-1\right) /\left(C^{2}+1\right)$ given by

$P\left(\mathrm{~N}_{\mathrm{cp}}=n\right)= \begin{cases}\sum_{i=1}^{n} \frac{\sigma^{i}}{i !} e^{-\sigma}\left(\begin{array}{c}n-1 \\ i-1\end{array}\right) \tau^{i}(1-\tau)^{n-i}, & \text { if } n \geq 1, \\ e^{-\sigma}, & \text { if } n=0\end{cases}$

where $\mathrm{N}_{\mathrm{cp}}$ is a Compound Poisson $\mathrm{rv}$ of the number of events per unit time corresponding to a stationary GE-type interevent rv.

The GE distribution is versatile, possessing pseudo-memoryless properties which make the solution of many GE-type queueing systems and networks analytically tractable (e.g., Kouvatsos [16]). Moreover, it has been experimentally established that the GE model, due to its extremal nature, defines pessimistic performance bounds on typical performance measures over corresponding estimates based on two-phase distributions having the same first two moments as the GE. The GE distribution is completely characterised in terms of mean rate, $v$ and, SCV, $C^{2}$ and it can be interpreted as an ME solution (c.f., Jaynes [7]), subject to the constraints of normalisation, discrete-time zero probability and expected value. In this sense, it can be viewed as the least biased distribution estimate, given the available information in terms of the constraints.

For $C^{2}<1$, the GE distributional model (with $\mathrm{F}(0)<1$ ) cannot be physically interpreted as a stochastic model. However, it can be meaningfully considered as a pseudo-distribution function of a flow model approximation of an underlying stochastic model in which negative branching pseudo-probabilities (or weights) are permitted. To this end, all analytical GE-type 
exact and approximate results obtained for queueing systems and networks when $C^{2}<1$ can also be used - by analogy - as useful heuristic approximations when $C^{2}<1$ as long as they satisfy basic queueing theoretic constraints (c.f. [16]). Note that utility of other improper twophase type distributions (with $C^{2}<1$ ) in the field of systems modelling has been proposed by various authors (e.g., Nojo and Watanabe [21], Sauer [23]).

\section{ME ANALYSIS OF A SHARED BUFFER QUEUE}

Consider a general queueing model of a shared buffer switching element with bursty arrivals, depicted in Figure 2. The queueing model consists of $\mathrm{R}$ parallel single server queues, where $\mathrm{R}$ is the number of output ports. Each server represents an output port and each queue corresponds to the address queue for the output port. There are RxR bursty and heterogeneous GE-type interarrival streams of cells, $R$ (multiple) streams to each of $R$ input ports. Each stream has a mean overall arrival rate, $\Lambda_{\mathrm{ji}}$, of cells and a SCV of interarrival time, $\mathrm{Ca}_{j i}^{2}$, for stream $(j, i), i, j=1,2, \ldots, \mathrm{R}$ (n.b., subscript $j$ is dropped in the case of a single stream per input port). Similarly, the transmission (or service) time of a cell at queue $i$ follows a GE distribution with mean rate $\mu_{i}$, and $\operatorname{SCV} C s_{i}^{2}$, for stream $i, i=1,2, \ldots, \mathrm{R}$. Let $\mathrm{K}$ be the size of the total shared buffer. A cell is lost if it arrives at a time when there is a total of $\mathrm{K}$ cells in the $\mathrm{R}$ queues. Without loss of generality, it is assumed that any of the $\mathrm{R}$ queues may attain the maximum size K.

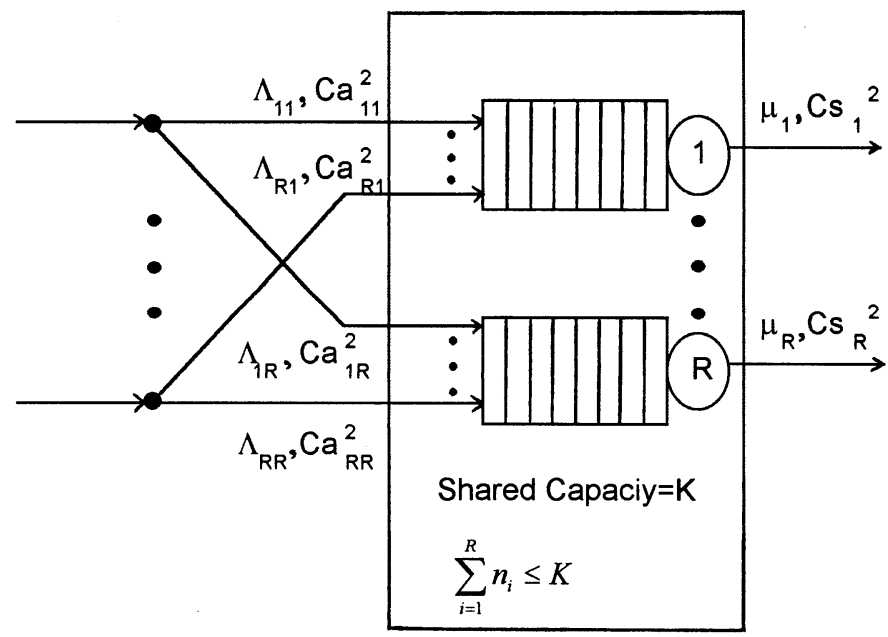

Figure 2 The $\mathrm{S}_{\mathrm{RxR}}\left(\mathrm{GE}^{\mathrm{R}} / \mathrm{GE} / 1 / \mathrm{K}\right)$ queueing model of a shared buffer switch. 
The queueing model of the shared buffer switching element is denoted by $\mathrm{S}_{\mathrm{R} \times \mathrm{R}}\left(\mathrm{GE}^{\mathrm{R}} / \mathrm{GE} / 1 / \mathrm{K}\right)$, such that

1. The overall interarrival times and service times at an $R \times R$ shared buffer queue are heterogeneous and GE distributed,

2. Each output port has a single server,

3. The total shared buffer capacity of the switch is $\mathrm{K}$.

Moreover, let the state of the system at any given time be represented by a vector $\mathbf{n}=\left(\mathrm{n}_{1}, \mathrm{n}_{2} \cdots, \mathrm{n}_{\mathrm{R}}\right)$, where $n_{i}$ is the number of cells in queue $\mathrm{i}, \mathrm{i}=1,2, \ldots, \mathrm{R}$, and

$$
\mathbf{n} \in \mathrm{S}(\mathrm{K}, \mathrm{R})=\left\{\mathbf{n}=\left(\mathrm{n}_{1}, \mathrm{n}_{2}, \ldots, \mathrm{n}_{\mathrm{R}}\right): \sum_{\mathrm{i}=1}^{\mathrm{R}} \mathrm{n}_{\mathrm{i}} \leq \mathrm{K}, 0 \leq \mathrm{n}_{\mathrm{i}} \leq \mathrm{K}, \mathrm{i}=1, \ldots, \mathrm{R}\right\} .
$$

Also let $\mathrm{p}(\mathbf{n}), \mathbf{n} \in \mathrm{S}(\mathrm{K}, \mathbf{R})$, be the joint state probability distribution.

Note that the ME solution of the $S_{R \times R}\left(G E^{R} / G E / 1\right) / K$ queueing system, $p(n)$ is of the same form as the ME solution of an $\mathrm{S}_{\mathrm{RxR}}(\mathrm{GE} / \mathrm{GE} / 1) / \mathrm{K}$ queueing system with a single (merged) arrival stream at each of the $R$ input points (c.f. Kouvatsos[14]), subject to a common set of mean value constraints. Both solutions are presented below.

\subsection{An ME Solution for the $S_{R x R}(G E / G E / 1) / K$ Queueing System: an Outline}

The form of the $\mathrm{ME}$ solution of an $\mathrm{S}_{\mathrm{RxR}}(\mathrm{G} / \mathrm{G} / 1) / \mathrm{K}$ queueing system, subject to normalisation and the constraints: server utilisation, $U_{i}, 0<U_{i}<1 ; \mathrm{MQL} L_{i}, U_{i} \leq L_{i}<K$; conditional aggregate probability $\varphi_{i}$ of a full buffer subject to $0<\varphi_{i}<1, n_{i}>0$, $i=1,2, \ldots, \mathrm{R}$, is given by the method of Largrange's undetermined multipliers as (c.f. (2.4))

$$
\mathrm{p}(\mathbf{n})=\frac{1}{Z} \prod_{i=1}^{\mathrm{R}} g_{i}^{\mathrm{s}_{i}(\mathbf{n})} x_{i}{ }^{n_{i}} y_{i}^{\mathrm{f}_{i}(\mathbf{n})}, \quad \forall \mathbf{n} \in \mathrm{S}(\mathrm{K}, \mathbf{R}),
$$

where $Z$ is the normalising constant

$$
Z=\sum_{n \in S(K, R)} \prod_{i=1}^{R} g_{i}^{s_{i}(n)} x_{i}^{n_{i}} y_{i}^{f_{i}(n)},
$$

$\mathrm{s}_{i}(\mathbf{n})$ and $\mathrm{f}_{i}(\mathbf{n})$ are auxiliary (indicator) functions defined by

$$
\mathrm{s}_{i}(\mathbf{n})= \begin{cases}1, & n_{i}>0 \\ 0, & \text { otherwise }\end{cases}
$$


$\mathrm{f}_{\mathrm{i}}(\mathbf{n})= \begin{cases}1, & \sum_{\mathrm{j}=1}^{\mathrm{R}} n_{j}=\mathrm{K} \wedge \mathrm{s}_{i}(\mathbf{n})=1, \\ 0, & \text { otherwise }\end{cases}$

and $\left\{g_{i}, x_{i}, y_{i}: i=1,2, \ldots, \mathrm{R}\right\}$ are the GE-type Largrangian coefficients corresponding to the constraints $\left\{U_{i}, L_{i}, \varphi_{i}: i=1,2, \ldots, \mathrm{R}\right\}$, respectively.

Lagrangian coefficients $\left\{g_{i}, x_{i}: i=1,2, \ldots, \mathrm{R}\right\}$ are obtained by making asymptotic connections with the ME solution of a stable GE/GE/1 queue (c.f.,[11]), namely

$g_{i}=\frac{\rho_{i}\left(1-x_{i}\right)}{x_{i}\left(1-\rho_{i}\right)}, \quad x_{i}=\frac{L_{i}-\dot{\rho}_{i}}{L_{i}}, \quad \rho_{i}=\Lambda_{i} / \mu_{i}, \quad i=1,2, \ldots, \mathrm{R}$,

where $L_{i}=\frac{\rho_{i}}{2}\left(1+\frac{C a_{i}^{2}+\rho_{i}^{2} C s_{i}^{2}}{1-\rho_{i}}\right), i=1,2, \ldots, \mathrm{R}$,

(i.e., $g_{i}$ and $x_{i}$ are assumed to be invariant to the buffer size K).

Moreover, Lagrangian coefficients $\left\{y_{i}: i=1,2, \ldots, \mathrm{R}\right\}$ can be computed by

1. Focusing on the flow balance equations

$$
\Lambda_{i}\left(1-\pi_{i}\right)=\mu_{i} U_{i}, \quad i=1,2, \ldots, \mathrm{R},
$$

where $\pi_{i}$ is the cell loss probability for an attempted arrival to the output port queue $i$,

2. Deriving recursive expressions for $\pi_{i}$ and $U_{i}, 1,2, \ldots, \mathrm{R}$, and

3. Solving numerically the resultant non-linear simultaneous equations, (n.b., for $R=2$, these equations can be solved analytically - see formulae (4.18)).

The normalising constant can be determined by applying the generating function approach and can be computed recursively by [14]

$Z=\sum_{v=0}^{\mathrm{K}-1} C_{1}(v)+C_{2}(\mathrm{~K})$

where $\left\{C_{1}(v): v=0,1, \ldots, \mathrm{K}-1\right\}$ and $\left\{C_{2}(K)\right\}$ are determined via the following recursive formulae:

$C_{1}(v)=C_{1 R}(v), \quad v=0,1, \ldots, \mathrm{K}-1$, $C_{2}(\mathrm{~K})=C_{2 R}(\mathrm{~K})$, 
where

$C_{k r}(v)=C_{k, r-1}(v)-\left(1-B_{k, r}\right) x_{r} C_{k, r-1}(v-1)+x_{r} C_{k r}(v-1)$,

$B_{k, r}= \begin{cases}g_{r}, & k=1, \\ g_{r} y_{r}, & k=2,\end{cases}$

for $k=1,2, r=2, \ldots, R, v=1,2, \ldots, \mathrm{K}-2+k$, with initial conditions

$C_{k 1}(v)= \begin{cases}1, & v=0, \\ B_{k, 1} x_{1}{ }^{v}, & v=1,2, \ldots, N-2+k,\end{cases}$

$C_{k r}(0)=1 \quad r=2, \ldots, \mathrm{R}$,

for $\quad k=1,2$.

Similarly, the utilisation $\mathrm{U}_{\mathrm{i}}$ can be expressed as

$U_{i}=\frac{1}{Z}\left(\sum_{v=1}^{\mathrm{K}-1} C_{1}^{(i)}(v)+C_{2}^{(i)}(\mathrm{K})\right), \quad i=1,2, \ldots, \mathrm{R}$,

where

$C_{k}^{(i)}(v)=\left(1-B_{k, i}\right) x_{i} C_{k}^{(i)}(v-1)+B_{k, i} x_{i} C_{k}(v-1), \quad v=2, \ldots, K-2+k$,

$k=1,2, \quad i=1,2, \ldots, R$, with initial conditions $C_{k}^{(i)}(1)=B_{k, i} x_{i}$.

The marginal state probabilities $\left\{\mathrm{p}_{i}\left(\ell_{i}\right): \ell_{i}=0, . ., \mathrm{K}\right\}$ can be determined by using ME solution (4.1) and the recursive expressions for $C_{k}^{(i)}(v)$. Let $n(i)$ be the random variable for the number of cells at queue $i, i=1,2, \ldots, R$. Then the marginal state probabilities are given by (c.f. [14]).

$\mathrm{p}_{i}\left(\ell_{i}\right)=\operatorname{Pr}\left[n(i) \geq \ell_{i}\right]-\operatorname{Pr}\left[n(i) \geq \ell_{i}+1\right]$,

where

$$
\begin{aligned}
& \operatorname{Pr}\left[n(i) \geq \ell_{i}\right]=\frac{x_{i}^{\ell_{i}-1}}{Z}\left(\sum_{v=\ell_{i}}^{\mathrm{K}-1} C_{1}^{(i)}\left(v-\ell_{i}+1\right)+C_{2}^{(i)}\left(\mathrm{K}-\ell_{i}+1\right)\right), \\
& i=1,2, \ldots, R, \ell_{i}=1,2, \ldots, \mathrm{K} .
\end{aligned}
$$


Finally the aggregate state probabilities $\{\mathrm{p}(n): n=0, . ., \mathrm{K}\}$ are given by

$$
p(n)= \begin{cases}\frac{1}{Z} & n=0, \\ \frac{1}{Z} C_{1}(n), & n=1,2, \ldots, \mathrm{K}-1, \\ \frac{1}{Z} C_{21}(n), & n=\mathrm{K} .\end{cases}
$$

\subsection{An ME Solution for the $S_{R \times R}\left(G^{R} / G E / 1\right) / K$ Queueing System: An Extension}

Earlier applications of entropy maximisation (e.g., $[10,12,13,17])$ on arbitrary QNMs and shared buffer queues imply a decomposition into individual queueing systems with revised GE or GGeo-type interarrival and service time processes. These processes utilise analytic functions describing GE or GGeo-type flows amongst the queues of the network. Flows are split when going to different destinations and merged when converging from different sources. The formulae used to split flows are exact in the case of random routing. For GE or GGeo merging flows a two moment matching function is used to approximate the resultant stream with a GE or GGeo-type stream. This last operation may lead to some inaccuracies in extremal cases, where there are large differences in the size of the SCVs of the merging flows.

In this work, a ME QLD is proposed for an $\mathrm{S}_{\mathrm{RxR}}\left(\mathrm{GE}^{\mathrm{R}} / \mathrm{GE} / 1\right) / \mathrm{K}$ queueing system which employs multiple input streams. This ME solution is of the same form as (4.1), subject to mean value constraints $\left\{U_{i}, L_{i}, \varphi_{i}: i=1,2, \ldots, \mathrm{R}\right\}$. The Lagrangian coefficients $g_{i}$ and $x_{i}$ of ME solution (4.1) are assumed to be invariant of the buffer size and are thus of the same form as these of a stable $\mathrm{GE}^{\mathrm{R}} / \mathrm{GE} / 1$ queue (see Appendix I) i.e., $\left\{g_{i}, x_{i}: i=1,2, \ldots, \mathrm{R}\right\}$ are determined by making asymptotic connections with the ME solution of a stable $\mathrm{GE}^{\mathrm{R}} / \mathrm{GE} / 1$ queue and, clearly, are given by

$g_{i}=\frac{\rho_{i}\left(1-x_{i}\right)}{x_{i}\left(1-\rho_{i}\right)}, \quad x_{i}=\frac{\widetilde{L}_{i}-\rho_{i}}{\widetilde{L}_{i}}, \quad i=1,2, \ldots, \mathrm{R}$,

where $\widetilde{L}_{i}$ is the MQL of a stable $\mathrm{GE}^{\mathrm{R}} / \mathrm{GE} / 1$ queue (see Appendix I) and is given by

$$
\tilde{L}_{i}=\frac{1}{2}\left(\rho_{i}+\frac{\sum_{j=1}^{R} \rho_{j i} C a_{j i}^{2}+\rho_{i}^{2} C s_{i}^{2}}{1-\rho_{i}}\right),
$$

with $C a_{j i}^{2}$ the SCV of stream i, $\rho_{j i}=\Lambda_{j i} / \mu_{i}, \quad j=1,2, \ldots, \mathrm{R}$ and $\rho_{i}=\sum_{j=1}^{R} \rho_{j i}$. 
Equating $g_{i}$ and $x_{i}$ of the ME solution of a stable $\mathrm{GE}^{\mathrm{R}} / \mathrm{GE} / 1$ queue with those of a stable GE/GE/1 queue with overall (merged) interarrival parameters $\Lambda_{i}$ and $C a_{i}^{2}$, the following relationship can be established:

$$
\Lambda_{i} C a_{i}^{2}=\sum_{j=1}^{\mathrm{R}} \Lambda_{j i} C a_{j i}^{2}, \quad i=1,2, \ldots, \mathrm{R} .
$$

Thus, the ME solution of a stable $\mathrm{GE}^{\mathrm{R}}$ / GE / 1 queue can be considered as an ME solution of a stable $\mathrm{GE} / \mathrm{GE} / 1$ with merged arrival processes having as parameters

$$
\Lambda_{i}=\sum_{j=1}^{\mathrm{R}} \Lambda_{j i}, \quad i=1,2, \ldots, \mathrm{R}
$$

and

$$
C a_{i}^{2}=\sum_{j=1}^{\mathrm{R}} \frac{\Lambda_{j i}}{\Lambda_{i}} C a_{j i}^{2}, \quad i=1,2, \ldots, \mathrm{R} .
$$

Note that expressions (4.11) and (4.12) turn out to be identical with those sugested in by Gelenbe and Pujolle [3]. Moreover, the interdeparture process of a stable $\mathrm{GE}^{\mathrm{R}} / \mathrm{GE} / \mathrm{lqueue}$ has a SCV given by (c.f. $[10,16])$

$$
C d_{i}^{2}=\rho_{i}\left(1-\rho_{i}\right)+\rho_{i}^{2} C_{s i}^{2}+\left(1-\rho_{i}\right) C a_{i}^{2}, \quad i=1,2, \ldots, \mathrm{R}
$$

Let $\left\{\pi_{j i}: i, j=1, \ldots, \mathrm{R}\right\}$ be the CLPs of input streams $\{j\}$ at output ports $\{i\}$ of a shared buffer $\mathrm{S}_{\mathrm{RxR}}\left(\mathrm{GE}^{\mathrm{R}} / \mathrm{GE} / 1\right) / \mathrm{K}$ queue. These probabilities can be obtained by using similar GE-type arguments as those applied in the case of the shared buffer $S_{\mathrm{RxR}}(\mathrm{GE} / \mathrm{GE} / 1) / \mathrm{K}$ queue (c.f. [14]) and are given by

$$
\pi_{j i}=\frac{1}{Z}\left(F_{j i}(\mathrm{~K})+C_{2}(\mathrm{~K})\right)
$$

where $(i, j), i, j=1,2, \ldots R$, is the $j$ th flow to output port $i$, and

$$
F_{j i}(\mathrm{~K})=\delta_{j i} \sum_{v=0}^{\mathrm{K}-1} C_{1}(v)\left(1-\sigma_{j i}\right)^{\mathrm{K}-v}+\left(1-\delta_{j i}\right) \sum_{v=1}^{\mathrm{K}-1} C_{1}^{(i)}(v)\left(1-\sigma_{j i}\right)^{\mathrm{K}-v},
$$

where

$$
\delta_{j i}=\frac{r_{s i}}{r_{s i}\left(1-\sigma_{j i}\right)+\sigma_{j i}},
$$

with 
$r_{s i}=\frac{2}{C s_{i}^{2}+1}, \quad \sigma_{j i}=\frac{2}{C a_{j i}^{2}+1}, \quad \mathrm{i}, \mathrm{j}=1,2, \ldots \mathrm{R}, \quad \mathrm{K} \geq 2$.

Lagrangian coefficients $\left\{y_{i}: i=1,2, \ldots, \mathrm{R}\right\}$ of the $\mathrm{S}_{\mathrm{RxR}}\left(\mathrm{GE}^{\mathrm{R}} / \mathrm{GE} / 1\right) / \mathrm{K}$ can be determined by using the flow balance conditions,

$\sum_{j=1}^{R} \Lambda_{j i}\left(1-\pi_{j i}\right)=U_{i} \mu_{i}, \quad i=1,2, \ldots \mathrm{R}$.

Substituting (4.14) into (4.16) the following system of $R$ non-linear equations with $R$ unknowns $\left\{y_{i}: i=1,2, \ldots, \mathrm{R}\right\}$, is obtained:

$C_{2}^{(i)}(K)=\rho_{i} \sum_{v=1}^{K=1} C_{1}(v)-\sum_{j=1}^{R} \rho_{i j} F_{i j}(K)-\sum_{v=1}^{K-1} C_{1}^{(i)}(v)$

for all $i=1,2, \ldots, \mathrm{R}$ and $\mathrm{K} \geq 2$.

System (4.17) can be solved by applying the numerical algorithm of Newton-Raphson, which is generally expected to give quadratic convergence. One significant limitation of this method is the requirement that the partial derivatives of the Jacobian matrix must be calculated at each iteration. However, this requirement may be avoided by applying an efficient recursive scheme suggested in [14]. Thus, because of the recursive nature of the $\mathrm{z}$-transforms which are used in the computational implementation of the ME solution, the $S_{\mathrm{RxR}}\left(\mathrm{GE}^{\mathrm{R}} / \mathrm{GE} / 1 / \mathrm{K}\right)$ queueing model can be used as an effective building block in the analysis of large MINs.

Note that in the special case of $\mathrm{R}=2$, these equations (4.17) can be solved analytically yielding the following closed-form expressions

$y_{1}=\frac{1}{2 g_{1} x_{1}}(\sqrt{A-B})$,

where

$$
\begin{aligned}
& A=\left[\frac{x_{1}^{K}-x_{2}^{K}}{x_{1}^{K-1}-x_{2}^{K-1}}\right]^{2}+\left[C_{2}^{(2)}(K)-C_{2}^{(1)}(K)\right]^{2}\left[\frac{x_{1}-x_{2}}{x_{1}^{K}-x_{2}^{K}}\right]^{2}+2\left[C_{2}^{(2)}(K)+C_{2}^{(1)}(K)\right] \frac{x_{1}-x_{2}}{x_{1}^{K-1}-x_{2}^{K-1}} \\
& B=\frac{x_{1}^{K}-x_{2}^{K}}{x_{1}^{K-1}-x_{2}^{K-1}}-\left[C_{2}^{(2)}(K)-C_{2}^{(1)}(K)\right] \frac{x_{1}-x_{2}}{x_{1}^{K}-x_{2}^{K}}, \quad \text { and } \\
& y_{2}=\frac{y_{1} g_{1} x_{1}\left(x_{1}^{K}-x_{2}^{K}\right)+\left(C_{2}^{(2)}(K)-C_{2}^{(1)}(K)\right)\left(x_{1}-x_{2}\right)}{g_{2} x_{2}\left(x_{1}^{K}-x_{2}^{K}\right)}
\end{aligned}
$$

Proofs of equations (4.18) and (4.19) are given is Appendix II. 


\section{ME ANALYSIS OF BANYAN MINs WITH ARBITRARY SWITCH SIZES}

Consider a packet-switched finite buffered ATM switch with a Banyan MIN-based architecture depicted in Figure 3. The ATM switch consists of L levels and $M$ stages and employs as basic building blocks R-input and R-output shared buffer switching elements (RxR crossbar switches).

Let switch-(l,m) denoting a switching element located at the lth level and $m$ th stage of the MIN. Each output (input) port is connected to a ouput (input) pin. The input and output pins of each switching element are labelled (including the MIN's external input and output pins) as "input- $k$ " and "output- $k$ ", $k=0,1, \ldots, \mathrm{R}-1$ from top to bottom, respectively. In regular Banyan MINs, where all switching elements are the same size, $M=\log _{R} N$, where $R$ is the size of each switching element and $\mathrm{N}$ is the number of exteral inputs (or outputs). Regular Banyan MINs form an array of switching elements and in this case the number of switching elements in a row is referred to as the level $\mathrm{L}$, where $\mathrm{L}=\mathrm{N} / \mathrm{R}$.

The input/output ports of the MIN form an array of 'pins' which are indexed by a row then column. There are $\mathrm{N}$ pins at each stage. Each output pin is linked to a single down stream input pin at the next stage. Connections from output ports pins to input port pins can be made in an arbitrary way. These connections form the topology of the network and are represented in the forwards (FTM) and backwards (BTM) topology matrices. Note that in a Banyan MIN only one path exists between an external input pin and an external output pin. The FTM and BTM have both $\mathrm{M}$ columns and $\mathrm{N}$ rows representing the grid of output and input port pins, respectively. Element $(n, m)$ holds the number of the input \{output port pin at the $(m+1)^{\text {th }}$ $\{(\mathrm{m}-1)$ th $\}$ stage that is connected to output (input) port pin $\mathrm{n}$ at the $\mathrm{m}^{\text {th }}$ stage, respectively.

The traffic arriving at the external input pins of the MIN is assumed to be bursty and is represented by GE interarrival times. The service (transmission) times at the output ports are also assumed to be GE distributed with mean, $1 / \mu_{k}$ and SCV, $C s_{k}^{2}$. The flow to external input pin $\mathrm{k}$ is parameterised by the overall mean arrival rate, $\hat{\Lambda}_{k}$ and the SVC of interarrival times, $C a_{k}^{2}$. Incoming cells traverse the network according to both the network's topology matricies and $\left\{r_{k s}\right\}_{N x N}$, the routing probability matrix, where $r_{k s}$ is the probability that a cell originating at external input pin $\mathrm{k}$ has external output pin $\mathrm{s}$ as its destination. Cells arrive in geometrically distributed bulks, with an average bulk size of $\left(\mathrm{Ca}_{k}^{2}+1\right) / 2$. Cells that arrive in the same bulk will take the same route across the MIN i.e. the routing decission is made on a per bulk basis. It is assumed that stage 0 switching elements at the input edges of the MIN may have infinite or finite capacity buffers, $\left\{\mathrm{K}_{\ell 0}: \ell=0,1, ., \mathrm{L}-1\right\}$. Moreover, switching elements in the interior or last stage of the MIN each have a fixed finite capacity buffer,

$\left\{\mathrm{K}_{\ell \mathrm{m}}: \mathrm{m}=1,2, . ., \mathrm{M}-1 ; \ell=0,1, . ., \mathrm{L}-1\right\}$. A cell is lost if on arrival at a stage 0 switching element finds a full buffer. However, every cell that enters the MIN is guaranteed delivery to its destination. This constraint along with the finite buffers of internal switches, implies that the MIN internally operates a blocking mechanism, which in this paper is based on RS blocking (c.f., Introduction). 


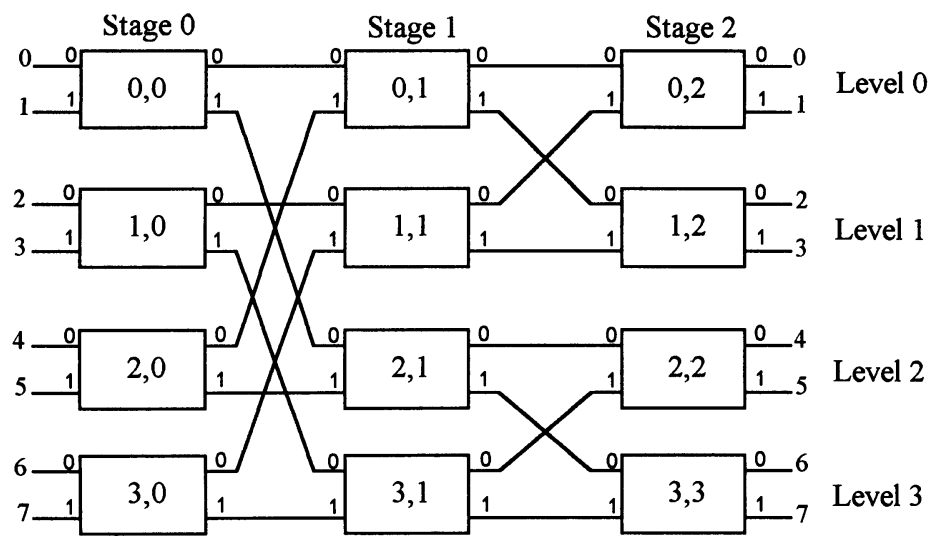

Figure 3 A 8x8 configuration of a regular Banyan Network

\subsection{A ME Product Form Approximation}

Suppose at any given time, the joint state of the network is denoted by $\mathbf{n}=\left(\mathbf{n}_{11}, \ldots, \mathbf{n}_{L M}\right)$, where $\mathbf{n}_{\mathrm{ij}}=\left(\mathbf{n}_{\mathrm{ij} 1}, \mathbf{n}_{\mathrm{ij} 2}, \ldots \mathbf{n}_{\mathrm{ijR}}\right)$ is the joint state of shared buffer queueing model of the switch- $(i, j)$ and $n_{i j k}$ is the number of cells queueing for output port $k, k=1,2, \ldots, \mathrm{R}$. Moreover, let $\mathrm{p}(\mathrm{n})$ be at any given time the joint state probability of the network. The form of a ME solution, $p(n)$, of a Banyan MIN, subject to normalisation and the marginal constraints of shared buffer queueing systems used in Section 4, namely utilisation, $U_{i j k}, 0<U_{i j k}<1, \mathrm{MQL}, L_{i j k}, U_{i j k}<L_{i j k}<\mathrm{K} i j$, and conditional aggregate full buffer probability with $n_{i j k}>0, \varphi_{i j k}, 0<\varphi_{i j k}<1, j=1,2, \ldots, R$, $i=1,2, \ldots, L M$, is given - via the method of Lagrange's undetermined multipliers - as

$p(\mathbf{n})=\frac{1}{Z} \prod_{i=1}^{L} \prod_{j=1}^{M} \prod_{k=1}^{R} g_{i j k}^{s_{i j k}\left(\mathbf{n}_{i j}\right)} x_{i j k} n_{i j k} y_{i j k} f_{i j k}\left(\mathbf{n}_{i j}\right)$,

where $Z$ is the normalising constant and $\left\{g_{i j k}, x_{i j k}, y_{i j k}\right\}$, are the Lagrangian coefficients corresponding to constraints $\left\{U_{i j k}, L_{i j k}, \varphi_{i j k}\right\}$, respectively and $s_{i j k}\left(\mathbf{n}_{i j}\right)$ and $f_{i j k}\left(\mathbf{n}_{i j}\right)$ are appropriate indicator functions such that $s_{i j k}\left(\mathbf{n}_{i j}\right)=1$, if $n_{i j k}>0$, or 0 , otherwise and $f_{i j k}\left(\mathbf{n}_{i j}\right)=1$, if $\sum_{k=1}^{\mathrm{R}} n_{i j k} \leq K_{i j}$, or 0 , otherwise, $\mathrm{k}=1,2, \ldots \mathrm{R}$. The form of ME solution (5.1) clearly suggests a product form approximation, namely

$$
\mathrm{p}(\mathbf{n})=\prod_{i=1}^{L} \prod_{j=1}^{M} p_{i j}\left(\mathbf{n}_{i j}\right)
$$


where $\mathrm{p}_{i j}\left(\mathbf{n}_{i j}\right)$ is determined by the ME solution (4.1) of each shared buffer queueing model.

The ME solution (5.1) can be implemented computationally by decomposing the network into individual building blocks of shared buffer switches-(i,j) with modified arrival and service parameters which capture the characteristics of the Banyan MIN.

\subsection{Flow Through the Switching Elements of a Banyan MIN}

The flow rate from each input pin through to each output pin of a Banyan network is calculated from the flow rate entering each input pin and the routing probability matrix $\left\{r_{k s}\right\}_{N x N}$. Let $\hat{\lambda}_{k s}$ be the effective flow rate from external input pin $\mathrm{k}$ to external output pin $\mathrm{s}$. Then, it follows that

$$
\hat{\lambda}_{k s}=\hat{\Lambda}_{k}\left(1-\pi_{k}\right) r_{k s} \quad k, s=0,1, . ., \mathrm{N}-1
$$

where $\pi_{k}=\pi_{a i}$, $\mathrm{i}$ is the input port of a switch at stage 0 that corresponds to input pin $\mathrm{k}$ and $\pi_{a i}$ is the aggregate CLP of input port i, i.e., the probability that an arriving cell via external input $\mathrm{k}$ will be turned away (c.f. Section 6.1).

In Banyan networks only one path exists between $\mathrm{k}$ and $\mathrm{s}$, so $\hat{\lambda}_{k s}$ is the contribution of flow given to each switch on the path from $\mathrm{k}$ to $\mathrm{s}$. The effective flow rates, $\left\{\lambda_{j i}\right\}$, across inputoutput pin pairs $\{(\mathrm{j}, \mathrm{i}): \mathrm{i}, \mathrm{j}=1,2, . ., \mathrm{R}\}$ of a switching element can be obtained by appropriate summation of flows $\left\{\hat{\lambda}_{k s}\right\}$. For each input pin $\mathrm{j}$, it is necessary to know the set of external input pins which connect to it (generally through other switches). Likewise, for each output pin $i$, it is necessary to know the set of external output pins which ultimately connect to it. Let these sets be denoted by $\operatorname{Inpins}(j, m)$ and $\operatorname{Outpins}(i, m)$, where $(j, m)$ and $(i, m)$ represent input pin $j$ of a switching element and output pin $i$ both at stage $m$, respectively. Any path that originates from an input pin in $\operatorname{Inpins}(j, m)$ and terminates at a output pin in Outpins(i,m) must pass through input $j$ to output $i$. Thus, the effective flow rate from input pin $j$ to output pin $i$, $\lambda_{j i}$, is given by

$$
\lambda_{j i}=\sum_{\substack{k \in \operatorname{Inpins}(j, m) \\ s \in \operatorname{Outpins}(i, m)}} \hat{\lambda}_{k s}, \quad i, j=1, \ldots, \mathrm{R} .
$$

The method of calculating Inpins $(j, m)$ and Outpins $(i, m)$ is given is Appendix III.

Note that the shared buffer $S_{\mathrm{RxR}}\left(\mathrm{GE}^{\mathrm{R}} / \mathrm{GE} / 1\right) / \mathrm{K}$ queueing model and product form approximation (5.1) are applicable to the performance analysis of packet switched finite buffered MINs with arbitrary configuration. However, in this more general case, there are more than one paths through the MIN, connecting an external input pin with an external output pin, and thus, some form of routing description is needed, in addition, to specify the flow. 


\section{ME ANALYSIS OF SWITCHING ELEMENTS WITHING THE BANYAN NETWORK}

This section presents an approximate ME analysis of three types of shared buffer queueing models of switching elements within Banyan network, based on the $S_{\mathrm{RxR}}\left(\mathrm{GE}^{\mathrm{R}} / \mathrm{GE} / 1\right) / \mathrm{K}$ buliding block queue and GE-type flow formulae. Note that for presentational purposes, only subscripts for, input/output ports and related flow streams are denoted in this and subsequent section.

\subsection{Case 1: Switching Elements at the Input Edges of the Network}

When a switch is at the input edge of the Banyan network, the actual (overall) arrival parameters are known. However, due to potential RS blocking from second stage switching elements, the perceived (effective) service time (i.e., total transmission time experienced by each packet) has to be calculated. The effective service time can be expressed in terms of the blocking probabilities. A service completer which finds its downstream buffer full repeats its service. As each output port is connected to only one input pin of a downstream switching element, it is appropriate to calculate the effective service time in terms of the overall blocking probability that a service completer at output port queue $i$ experiences at its downstream queue switch. This overall blocking probability is clearly given by

$\pi_{c i}=\sum_{l=1}^{R} \pi_{k l} \frac{\Lambda_{k l}}{\tilde{\Lambda}_{k}}, \quad \tilde{\Lambda}_{k}=\sum_{l=1}^{R} \Lambda_{k l}, \quad i, k=1,2, \ldots, \mathrm{R}$,

where $\mathrm{k}$ is the input pin of a switching element at the next stage which is connected with output pin i (defined in FTM), $l$ is an output pin of the same element which is connected with $\mathrm{k}$ and $\Lambda_{k l}$ is the overall arrival rate from input pin $\mathrm{k}$ to output pin $l, l=1,2, \ldots, \mathrm{R}$.

By considering GE-type probabilistic arguments, the effective service time parameters can be expressed by (c.f., [12])

$\hat{\mu}_{i}=\mu_{i}\left(1-\pi_{c i}\right) \quad i=1,2, \ldots, \mathrm{R}$,

and

$$
\hat{C} s_{i}^{2}=\pi_{c i}+\left(1-\pi_{c i}\right) C s_{i}^{2}, \quad i=1,2, \ldots, \mathrm{R} .
$$

The arrival rate from the external input stream $\mathrm{j}$ to output pin $\mathrm{i}, \Lambda_{j i}$, is obtained by multiplying input rate, $\hat{\Lambda}_{j}$, by the sum of the appropriate routing probabilities (i.e. adding together the the probabilities from $j$ to all external (destination) output pins that pass through $i$ ), namely

$$
\Lambda_{j i}=\hat{\Lambda}_{j} \sum_{s \in O u t p i n s(i, 1)} r_{j s}, i, j=1,2, \ldots, \mathbf{R}
$$


Moreover, the SCV of the interarrival process from input stream $j$ to input pin $i$ is the same as that of the external SCV of interarrival time, as routing occurs on a per bulk basis (see Section 5) i.e.,

$C a_{j i}^{2}=C a_{j}^{2}, \quad i, j=1,2, \ldots, \mathrm{R}$

where $C a_{j}^{2}$ is the SCV of the overall interarrival time at external input pin i.

For first stage switching elements with infinite capacity, the SCV of the interdeparture process is clearly given by (cf., $(4.13)[10,12]$

$C d_{i}^{2}=\hat{\rho}_{i}\left(1-\hat{\rho}_{i}\right)+\hat{\rho}_{i}^{2} \hat{C} s_{i}^{2}+\left(1-\hat{\rho}_{i}\right) C a_{i}^{2}, \quad i=1,2, \ldots, \mathrm{R}$,

where

$C a_{i}^{2}=\sum_{j=1}^{R} \frac{\Lambda_{j i}}{\Lambda_{i}} C a_{j i}^{2}, \quad$ and $\quad \hat{\rho}_{i}=\Lambda_{i} / \hat{\mu}_{i}, \quad i=1,2, \ldots, \mathrm{R}$.

Note that in this case, each output port behaves as if it were an independent $\mathrm{GE}^{\mathrm{R}} / \mathrm{GE} / 1$ queue with marginal ME QLD, $p_{r}\left(n_{r}\right), n_{r}=1,2, \ldots, \mathrm{K}$, given in Appendix I.

For first stage switching elements of finite capacity, the SCV of the interdeparture process is clearly given by (c.f., $(4.13),[10,12])$

$C d_{i}^{2}=\hat{\rho}_{i}\left(1-\hat{\rho}_{i}\right)+\hat{\rho}_{i}^{2} \hat{C} s_{i}^{2}+\left(1-\hat{\rho}_{i}\right) \hat{C} a_{i}^{2}, \quad i=1,2, \ldots, \mathrm{R}$,

where

$$
\begin{array}{lll}
\lambda_{j i}=\Lambda_{j i}\left(1-\pi_{j i}\right), & \hat{C} a_{j i}^{2}=\pi_{j i}+\left(1-\pi_{j i}\right) C a_{j i}^{2}, & i, j=1,2, \ldots, \mathrm{R}, \\
\lambda_{i}=\sum_{j=1}^{R} \lambda_{j i}, & \hat{C} a_{i}^{2}=\sum_{j=1}^{R} \frac{\lambda_{j i}}{\lambda_{j}} \hat{C} a_{j i}^{2} & i=1,2, \ldots, \mathrm{R},
\end{array}
$$

and

$\hat{\rho}_{i}=\lambda_{i} / \hat{\mu}_{i}, \quad i=1,2, \ldots, \mathrm{R}$,

The CLP $\pi_{j i}$ can be determined from the ME solution of the shared buffer $\mathrm{S}_{\mathrm{RxR}}\left(\mathrm{GE}^{\mathrm{R}} / \mathrm{GE} / 1\right) / \mathrm{K}$ queue (c.f., Section 4.2), namely, 


$$
\pi_{j i}=\frac{F_{j i}(K)+C_{2}(K)}{\sum_{v=0}^{K-1} C_{1}(v)+C_{2}(K)},
$$

where $\mathrm{F}_{\mathrm{ji}}(\mathrm{K})$ is given by equation (4.16) incorporating parameters $\Lambda_{j i}, C a_{j i}^{2}, \hat{\mu}_{i}, \hat{C} s_{i}^{2}$, as appropriate.

The aggregate blocking probability, $\pi_{a j}$, at input $\operatorname{pin} \mathrm{j}$ is clearly given by

$$
\pi_{a j}=\sum_{i=1}^{R} \pi_{j i} \frac{\Lambda_{j i}}{\Lambda_{j}}, j=1,2, . ., \mathrm{R} .
$$

Note that $C_{1}(v)$ is a function of the Larangian coefficients $\left\{g_{i}, x_{i}: i=1,2, \ldots, R\right\}$ (which can be calculated from the input parameters), whilst $C_{2}(\mathrm{~K})$ is dependent upon all Lagrangian coefficients $\left\{g_{i}, x_{i}, y_{i}: i=1,2, \ldots, R\right\}$. The $\left\{y_{i}\right\}$ coefficients are obtained by solving the nonlinear equations which are of the same form as the ones determined by (4.17), if $R>2$ or (4.18)(4.19), if $\mathrm{R}=2$. The solution of these equations along with those of Section 4.2 give the QLDs of switching elements at stage 0 of the MIN together with other performance metrics.

\subsection{Case 2: Switching Elements at the Interior of the Network}

When a switching element is internal to the Banyan network at stage $m, m=1,2, \ldots, M-1$, the throughput (effective arrival rate) can be determined in terms of the effective arrival rates of the external input ports, the routing probabilities and the network topology. The SCV of the effective interarrival process is obtained from the SCV of the output process of the previous stage. The values of the Lagrangian coefficients of the ME solution $\mathrm{p}(\mathbf{n}), \mathbf{n} \in S(N, R)$, can be computed in terms of parameters of the overall flow which are related to the parameters of the effective flows and the blocking probabilities. These form a set of additional equations to those in Section 6.1 which (in addition) need to be solved to produce the QLD and other performance metrics for each internal switching element.

Let the effective flow rate that enters an input pin $\mathrm{j}$ be denoted by $\lambda_{j}, \mathrm{j}=1,2, \ldots \mathrm{R}$, with its component flow $(\mathrm{j}, \mathrm{i})$ going to output port $\mathrm{i}$ be denoted by $\lambda_{j i}, \mathrm{i}=1,2, \ldots, \mathrm{R}$. Let $\hat{C} \alpha_{j i}^{2}$, $\mathrm{i}, \mathrm{j}=1,2, \ldots, \mathrm{R}$, be the SCV of flow $(\mathrm{j}, \mathrm{i})$ and $\pi_{j i}, \mathrm{i}, \mathrm{j}=1,2, \ldots, \mathrm{R}$ be the blocking probability that flow (j,i) will find a full buffer. Using these parameters, the overall flow from each input pin to each output port can be calculated, as follows:

The overall flow rate, $\Lambda_{j i}$, is clearly given by (c.f. [12]) 
$\Lambda_{j i}=\frac{\lambda_{j i}}{1-\pi_{j i}} \quad, \quad i, j=1,2, \ldots, \mathrm{R}$

and from the GE-type splitting flow formulae

$C a_{j i}^{2}=\frac{\hat{\mathrm{C}} a_{j i}^{2}-\pi_{j i}}{1-\pi_{j i}}, \quad j, i=1,2, \ldots, \mathrm{R}$,

where $\pi_{j i}$ is calculated from the ME solution of the shared buffer $S_{\mathrm{RxR}}\left(\mathrm{GE}^{\mathrm{R}} / \mathrm{GE} / 1\right) / \mathrm{K}$ queue as described in Section (6.1).

The total effective arrival rate at input pin $\mathrm{j}, \lambda_{j}$ is expressed as

$\lambda_{j}=\sum_{k=1}^{R} \lambda_{k j}, \quad j=1,2, \ldots, \mathrm{R}$,

whilst the transition probability of a job going from input pin $\mathrm{i}$ to output pin $\mathrm{j}$ is clearly given by

$\alpha_{j i}=\frac{\lambda_{j i}}{\lambda_{j}}, \quad i, j=1,2, \ldots, \mathrm{R}$.

Packets that arriving in the same batch follow the same route through the network. This means that within the network, splitting of departing flows (from individual servers) may be complex, but fall within two schemes. In the first scheme individual packets choose their own downstream queue, upon service completion, according to a Bernoulli filter. In the second scheme the routing decission is made on a per bulk basis where the head of the bulk (i.e. the first packet in the bulk) chooses its downstream queue according to a Bernoulli filter and subsequent members of the bulk follow in its path. The second scheme produces bigger arriving bulks that the first scheme. To this end, the effective SCV of the arrival process is determined from the GE-type splitting flow formulae, namely

$\hat{C} a_{j i}^{2}=1+\left(C d_{p r e d(j)}^{2}-1\right) \alpha_{j i}, i, j=1,2, \ldots, \mathrm{R}$,

where $C d_{p r e d(j)}^{2}$ is the SCV of the interdeparture process from the upstream port/switch connected at stage $m-1$ to input pin $j$ whose location is given by vector BTM (i,m), $i=1,2, \ldots, R$, $\mathrm{m}=1,2, \ldots, \mathrm{M}-1$.

If the protocol indicates that the entire departing bulk will be directed to the same destination input port, then no splitting takes place and

$\hat{C} a_{j i}^{2}=C^{2} \operatorname{pred}(j), j=1,2, \ldots, \mathbf{R}$. 
Finally, the interdeparture process from output port $i$ is given by (4.13), namely

$C d_{i}^{2}=\hat{\rho}_{i}\left(1-\hat{\rho}_{i}\right)+\hat{\rho}_{i}^{2} \hat{C} s_{i}+\left(1-\hat{\rho}_{i}\right) \hat{C} a_{i}^{2}, i=1,2, \ldots, \mathrm{R}$,

where

$$
\hat{C} a_{i}^{2}=\sum_{j=1}^{R} \frac{\lambda_{j i}}{\lambda_{i}} \hat{C} a_{j i}^{2} \text { and } \hat{\rho}_{i}=\lambda_{i} / \hat{\mu}_{i}, \quad i=1,2, \ldots, \mathrm{R} .
$$

As only the effective arrival parameters are known, the overall arrival parameters are given in terms of the blocking probabilities, which are themselves given by equation (4.14). These equations together form $\mathrm{RxR}$ non-linear simultaneous equations with $\mathrm{RxR}$ unknowns (i.e. the $\pi_{j i}$ 's). Writing these equations as functions of the $\pi_{j i}$ 's gives

$$
f_{j i}=\pi_{j i}-\frac{F_{j i}(K)+C_{2}(K)}{\sum_{v=0}^{K-1} C_{1}(v)+C_{2}(K)}, \quad i, j=1,2, \ldots, \mathrm{R}
$$

Assuming that the value of $C_{2}(\mathrm{~K})$ is known, the equations are solved using NewtonRaphson's method to give the value of $\pi_{j i}$ 's. After the $\pi_{j i}$ 's are calculated, the $\left\{y_{i}\right\}$ coefficients are obtained by solving the non-linear equations (4.17), if $\mathrm{R}>2$ or (4.18), if $\mathrm{R}=2$. In the case of $\mathrm{R}>2$, a new value for $C_{2}(K)$ is calculated. This process is repeated until there is no change is the value of $C_{2}(K)$. The solution of these equations along with those of Section 4.2 give the QLDs of switching elements internal to the MIN together with other performance metrics.

\subsection{Case 3: Switching at the Output Edges}

When a switching element is at the external edge of the MIN, then its performance analysis follow from the ME solution of the shared buffer $S_{R \times R}\left(G E^{R} / G E / 1\right) / K$ queueing model of an internal switching element, except that the mean rate and SCV of the service time of each output port $\mathrm{i}$ are, respectively, are the actual parameters $\left(\mu_{i}, C s_{i}^{2}\right), \mathrm{i}=1,2, \ldots, \mathrm{R}$.

\section{AN ME APPROXIMATION PROCEDURE FOR THE PERFORMANCE ANALYSIS OF BANYAN MINS}

In this section an approximate procedure for obtaining the ME QLDs and other performance metrics at each building block of a Banyan MIN based shared buffer ATM switch is described. The procedure for infinite and finite first stage building blocks differ only in that the later 
includes the calculation of the first stage blocking probabilities and the flow rates through the network which are depended upon these probabilities. It is assumed that and the interdeparture processes to be of GE type. When these processes split into a number of streams distributed, to different output ports, it is assumed that the splitting is Bernoulli. These assumptions give rise to interarrival processes which are approximately the superposition of GE streams. Thus interarrival processes can be determined and their parameters evaluated.

\subsection{An ME Algorithm the Analysis of Banyan Networks}

Begin

Step 1. Initialise all cell loss probabilities. Set SCV of inter-arrival times to 1;

Step 2. Calculate effective flows across Banyan MIN and at each switching element (c.f. section 5.2);

Step 3. At the first stage represent each of its switching elements as a shared buffer building block queue $S_{\mathrm{RxR}}\left(G E^{\mathrm{R}}\left(\Lambda_{j i}, C a_{j i}^{2}\right) / G E\left(\hat{\mu}_{i}, \hat{C} s_{i}^{2}\right) / 1\right) / \infty, i, j=i, 2, \ldots, \mathrm{R}$, in the case of infinite capacity, or as $S_{\mathrm{RxR}}\left(G E^{\mathrm{R}}\left(\Lambda_{j i}, C a_{j i}^{2}\right) / G E\left(\hat{\mu}_{i}, \hat{C} s_{i}^{2}\right) / \mathrm{l}\right) / \mathrm{K}, \quad i, j=1,2, \ldots, \mathrm{R}$ for the case of finite capacity, and calculate for each output pin i the SCV of the interdeparture process $C d_{i}^{2}, \mathrm{i}=1,2, \ldots, \mathrm{R}$, to be used in the next stage using equations (6.6) and (6.7), as appropriate;

Step 4. From left to right do until last but one stage:

represent each stage switching element as a shared buffer building block queue $S_{\mathrm{RxR}}\left(G E^{\mathrm{R}}\left(\lambda_{j i}, \hat{C} a_{j i}^{2}\right) / G E\left(\hat{\mu}_{i}, \hat{C} s_{i}^{2}\right) / 1\right) / \mathrm{K}, \quad i, j=1,2 \ldots, \mathrm{R}, \quad$ and calculate for each output pin $i$ the $\mathrm{SCV}$ of the interdeparture process $C d_{i}^{2}, i=1,2, \ldots \mathrm{R}$, for the next stage, using equation (6.14)

Step 5. Analyse the performance of each switching element by solving a shared buffer building block queue $S_{\mathrm{RxR}}\left(G E^{\mathrm{R}}\left(\lambda_{j i}, \hat{C} a_{j i}^{2}\right) / G E\left(\mu_{i}, C s_{i}^{2}\right) / 1\right) / \mathrm{K}, \quad i, j=1, \ldots, \mathrm{R}$.

For first stage switching elements with infinite capacity repeat Steps 3-5 and for the corresponding case of finite capacity repeat Steps 2-5 until convergence of the calculated values of the SCV of the interdeparture times and the blocking probabilities of the first stages (as appropriate). Print out ME QLDs and typical performance metrics.

End.

\section{Remarks}

The main computation effort of the ME algorithm is at every iteration between steps 3 and 5 . The non-linear system of equations, $\left\{y_{i}: i=1,2, \ldots, R\right\}$, for each switching element can be written as $Y=F(Y)$, where $Y$ and $F$ are column vectors of dimension $\Omega$, where $\Omega$ is the cardinality of the set $\left\{y_{i}\right\}$. Similarly the non-linear equations $\left\{\pi_{i j}: i, j=1,2, \ldots, R\right\}$ can be written as $\Pi=G(\Pi)$, where $\Pi$ and $G$ are column vectors of dimension $\Omega^{\prime}$, where $\Omega^{\prime}$ is the cardinality of the set $\left\{\pi_{\mathrm{ij}}\right\}$. It can be verified that the computational cost of the algorithm is $\mathrm{O}\left(\mathrm{ML}\left(\Omega^{3}+\Omega^{\prime 3}\right)\right)$, where $M$ is the number of stages and $L$ is the number of levels of the MIN, $\Omega^{3}$ is the number of 
manipulations for inverting the Jacobian of $F$ with respect to $Y$ and $\Omega^{\prime 3}$ is the number of manipulations for inverting the Jacobian of $G$ with respect to $\Pi$.

The existence and unicity for the solution of the system of non-linear equations is difficult to prove analytically due to the complexity of the expressions of the blocking probabilities $\left\{\pi_{\mathrm{ij}}\right\}$ and the expression of Lagranagian coefficients $\left\{\mathrm{y}_{\mathrm{i}}\right\}$. Furthermore no strict mathematical justification can be given for the convergence of $\left\{C d_{i}^{2}: i=1,2, \ldots, \mathrm{R}\right\}$; nevertheless, numerical instabilities or non-convergence have never been observed in many experiments that have been carried out. If, however, at some iteration it is observed for at least one queue $\mathrm{j}$ that $\hat{\rho}_{j}=\left(\lambda_{j} / \mu_{j}\right) \geq 1$, then there exists only one trivial solution with $\pi_{i j}=1, i \in\{1,2, \ldots, R\}$, which is outside the domain at validity of the model.

When switching elements of infinite capacity are present at the first stage 0 , necessary conditions for the stability of the entire network are not obvious due to the constraining influence on a output port's service rate by downstream blocking. In essence, the stability condition for a single output port is that the effective arrival rate be less than its effective service rate which can only be approximately determined. This subject merits further research.

In cases of hot spot routing, cells are directed towards one particular output with a high probability. As this probability approaches unity, the MIN becomes equivalent to an arbitrary network with blocking and has an inverted tree configuration.

\section{NUMERICAL RESULTS}

This section presents typical numerical results in Tables 1-12 focusing on $8 \times 8$ (c.f., Tables 111) and $27 \times 27$ (c.f., Table 12) Banyan MINs with $2 \times 2$ and $3 \times 3$ switching elements, respectively. The aims of the study is to (i) validate the relative accuracy of the ME approximation algorithm against simulation (SIM) (c.f., Tables 1-8) (ii) define experimental bounds (c.f., Table 12) and, (iii) perform a buffer capacity optimisation across the entire Banyan Network (c.f., Tables 9-11).

In all experiments, external input ports of the Banyan $\mathrm{MIN}$ at stage 0 receive traffic with identical parameters. In total, three different routing schemes are adopted, namely uniform routing (regular traffic) towards the external output pins at final stage 2 (c.f., Tables 1-3, 612 ), and moderately or substantially biased routing towards an external output pin referred to as a warm spot (c.f., Table 4) or hot spot (c.f., Table 5), respectively. Note that in the case of uniform routing all switching elements belong to a particular stage will have the same output statistics. However, in the general case of non-uniform routing, switching elements within a stage will have different performance metrics. For each input port at stage 0 , and without loss of generality, identical routing probabilities biased towards the warm or hot spot are used in Tables 4 and 5, respectively. As a consequence, switching elements at each stage of the decode tree (i.e., the tree composed from the routes connecting external input pins with the warm or hot spot external output port) will have identical performance metrics. Thus, in both cases of uniform and non-uniform routing, performance metrics are only reported once in Tables $1-12$ respectively. 
Tables 1-8 present a validation study of the ME algorithm against simulation which includes aggregate MQLs $\left\{L_{i}: i=0,1,2\right\}$ at stages 0,1 and 2 , throughputs $\left\{\lambda_{2}\right\}$ of either a typical external output port under uniform routing (c.f., Tables 1-3, 6-8) and warm/hot spot external output port (c.f., Tables 4 and 5), and also the aggregate CLP of a typical switching element at stage 0 . Moreover, Tables 6-8 display aggregate and marginal state probabilities for a typical $8 \times 8$ Banyan network under uniform quoting. Note that the simulation results in Tables 1-8 were produced at $95 \%$ confidence intervals by using the Queueing Network Analysis Package (QNAP-2). It can be observed that the ME solutions are consistently comparable with those of simulation (SIM) for a wide range of parameterisation, including deterministic transmission times applicable to ATM switching elements. Note that confidence intervals are of small magnitude e.g., typically \pm 0.01 for MQLs. Moreover, percentage differences for MQLs are generally less than $10 \%$ and error tolerances for state and blocking probabilities, (i.e., absolute differences between ME and SIM results) are less than 0.05. The accuracy of ME approximations begin to deteriorate as the value of SCVs increases. This can be attributed to further violation of renewality assumptions of the various flow in the network.

The ME algorithm is utilised in performing a buffer capacity assignment optimisation across the Banyan MIN (c.f. Tables 9-11). Given an overall buffer allocation for the entire network, it is possible to carry out buffer assignments to individual switching elements in order to optimize the throughput or the end-to-end delay. Three different buffer allocation policies are considered by assigning more of the allocated buffer capacity to the first, second and third stages, respectively. From Tables 9-11, it can be observed that by placing more of the buffer allocation at the first stage of the network, the throughput can be increased whilst the end-toend delay is not adversely affected. This behavoir is intuitively correct since the CLP is smaller than in the other two cases, thus allowing more packets into the network.

Finally, Table 12 focus on $27 \times 27$ Banyan networks with $3 \times 3$ switching elements under regular traffic. Relative performance comparisons are carried out between the ME solutions produced incorporating the routing of entire bulks within the network (c.f., (6.14)) and SIM results produced using specially designed programs written in $\mathrm{C}$. It can be seen that the analytic solutions for first stage MQL, $\left\{L_{0}\right\}$ and aggregate CLPs, $\left\{\pi_{\alpha}\right\}$, are comparable in accuracy to those of simulation, as in the examples of Tables 1-8 (n.b., both ME algorithm and simulations use identical external inputs at stage 0 ). However, the ME solutions define (experimentally) pessimistic bounds over the corresponding SIM results produced concerning the MQLs of output ports at stages 1 and 2. This behaviour is due to the fact that the ME approximation overestimates the size of the bulk transitions in the interior of the network, and, subsequently, the SCV of the interarrival time of each internal and last stage output port. The study of analytic performance bounds merits further research.

\section{CONCLUSIONS}

A cost-effective approximate algorithm, based on the principle of $\mathrm{ME}$ and the notion of system decomposition, is proposed for the performance analysis and prediction of packet-switched buffered Banyan MIN-based ATM switch architectures with arbitrary buffer and building block sizes, GE-type interarrival and service times and RS internal blocking. Analytic ME solutions 
Table 1 Uniform Routing

\begin{tabular}{|c|c|c|c|c|c|c|c|c|c|}
\hline \multirow[t]{2}{*}{$\begin{array}{c}\text { Banyan } \\
\text { MIN } \\
\text { No. }\end{array}$} & \multicolumn{3}{|c|}{$\begin{array}{c}\text { Input Data } \\
\left\{r_{i j}=0.125, i, j=0,1, \ldots, 7\right\} \\
N=8 ; \mu=1 ; K=9\end{array}$} & \multicolumn{6}{|c|}{ Output Statistics } \\
\hline & $\Lambda$ & $\mathrm{Ca}^{2}$ & $\mathrm{Cs}^{2}$ & $\mathrm{~L}_{0}$ & $\mathrm{~L}_{1}$ & $\mathrm{~L}_{2}$ & $\lambda_{2}$ & $\pi_{\alpha}$ & Method \\
\hline 1 & 0.5 & 3 & 3 & $\begin{array}{l}3.1760 \\
3.1100\end{array}$ & $\begin{array}{l}2.6261 \\
2.7210\end{array}$ & $\begin{array}{l}2.3536 \\
2.3530\end{array}$ & $\begin{array}{l}0.4387 \\
0.4440\end{array}$ & $\begin{array}{l}0.1226 \\
0.1211\end{array}$ & $\begin{array}{l}\text { ME } \\
\text { SIM }\end{array}$ \\
\hline 2 & 0.5 & 5 & 5 & $\begin{array}{l}3.4429 \\
3.4620\end{array}$ & $\begin{array}{l}2.7746 \\
2.9050\end{array}$ & $\begin{array}{l}2.3691 \\
2.3300\end{array}$ & $\begin{array}{l}0.3869 \\
0.3877\end{array}$ & $\begin{array}{l}0.2263 \\
0.2229\end{array}$ & $\begin{array}{l}\text { ME } \\
\text { SIM }\end{array}$ \\
\hline 3 & 0.5 & 7 & 7 & $\begin{array}{l}3.5240 \\
3.6990\end{array}$ & $\begin{array}{r}2.8164 \\
2.9180\end{array}$ & $\begin{array}{l}2.3376 \\
2.3020\end{array}$ & $\begin{array}{l}0.3502 \\
0.3466\end{array}$ & $\begin{array}{l}0.2995 \\
0.3020\end{array}$ & $\begin{array}{l}\text { ME } \\
\text { SIM }\end{array}$ \\
\hline 4 & 0.5 & 11 & 11 & $\begin{array}{l}3.5525 \\
4.0030\end{array}$ & $\begin{array}{l}2.8112 \\
2.8840\end{array}$ & $\begin{array}{l}2.2586 \\
2.0940\end{array}$ & $\begin{array}{l}0.3020 \\
0.2912\end{array}$ & $\begin{array}{l}0.3959 \\
0.4175\end{array}$ & $\begin{array}{l}\text { ME } \\
\text { SIM }\end{array}$ \\
\hline 5 & 0.5 & 15 & 15 & $\begin{array}{l}3.5372 \\
4.1590\end{array}$ & $\begin{array}{l}2.7735 \\
2.8510\end{array}$ & $\begin{array}{l}2.1887 \\
1.9650\end{array}$ & $\begin{array}{l}0.2711 \\
0.2559\end{array}$ & $\begin{array}{l}0.4578 \\
0.4862\end{array}$ & $\begin{array}{l}\text { ME } \\
\text { SIM }\end{array}$ \\
\hline
\end{tabular}

for the QLD of a shared buffer $\mathrm{S}_{\mathrm{RXR}}\left(\mathrm{GE}^{\mathrm{R}} / \mathrm{GE} / 1\right) / \mathrm{K}$ queue in conjunction with GE-type formulae for the first two moments of the effective service times and traffic flows in the network, play the rôle of effective building blocks in the decomposition process of the entire network. Numerical results are included to illustrate the relative accuracy of $\mathrm{ME}$ approximations against simulation, define experimental MQL bounds in the interior and last stage of the network and to investigate the buffer capacity optimisation across the entire MIN. This study has shown that the ME approximation algorithm is a credible analytic tool for the cost- effective performance modelling and optimisation of complex MINs represented by Banyan networks. The ME algorithm can be extended towards the approximate analysis of ATM switch architectures with space and service priorities. Moreover, closed form expressions for queueing models of ATM networks with both bursty and correlated traffic can be derived based on the stochastic analysis of single finite queues with batch renewal arrival processes (c.f.,[18]). Extensions of this kind are the subject of current study. 
Table 2 Uniform Routing

\begin{tabular}{|c|c|c|c|c|c|c|c|c|c|}
\hline $\begin{array}{c}\text { Banyan } \\
\text { No }\end{array}$ & $\left\{\begin{array}{r}\left\langle r_{i j}=0\right. \\
N\end{array}\right.$ & $\begin{array}{l}\text { ut } D \\
\text {,i,j= } \\
\mu=1\end{array}$ & $\begin{array}{l}, \ldots, 7\} ; \\
=9\end{array}$ & & & Output & Statistics & & \\
\hline \multirow{3}{*}{10} & $\Lambda$ & $\mathrm{Ca}^{2}$ & $\mathrm{Cs}^{2}$ & $\mathrm{~L}_{0}$ & $\mathrm{~L}_{1}$ & $\mathrm{~L}_{2}$ & $\mathrm{~L}_{2}$ & $\lambda_{\alpha}$ & Method \\
\hline & \multirow[t]{2}{*}{0.3} & \multirow[t]{2}{*}{7} & \multirow[t]{2}{*}{7} & 2.1278 & 1.6379 & 1.3560 & 0.2451 & 0.1829 & ME \\
\hline & & & & 1.9580 & 1.5800 & 1.3510 & 0.2537 & 0.1497 & SIM \\
\hline \multirow[t]{2}{*}{11} & \multirow[t]{2}{*}{0.5} & \multirow[t]{2}{*}{7} & \multirow[t]{2}{*}{7} & 3.5240 & 2.8164 & 2.3376 & 0.3502 & 0.2995 & ME \\
\hline & & & & 3.6990 & 2.9400 & 2.2480 & 0.2467 & 0.3020 & SIM \\
\hline \multirow[t]{2}{*}{12} & \multirow[t]{2}{*}{0.7} & \multirow[t]{2}{*}{7} & \multirow[t]{2}{*}{7} & 4.7570 & 3.7642 & 2.9907 & 0.4081 & 0.4169 & $\mathrm{ME}$ \\
\hline & & & & 5.2130 & 3.7750 & 2.6850 & 0.3847 & 0.4502 & SIM \\
\hline
\end{tabular}

Table 3 Uniform Routing

\begin{tabular}{|c|c|c|c|c|c|c|c|c|c|}
\hline $\begin{array}{c}\text { Banyan } \\
\text { No. }\end{array}$ & $\begin{array}{r}\left\{r_{i j}=0 .\right. \\
N\end{array}$ & $\begin{array}{l}\text { put Dc } \\
5, i, j= \\
; \mu=1 \text {; }\end{array}$ & 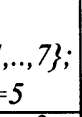 & & & Output S & tatistics & & \\
\hline \multirow{3}{*}{13} & $\Lambda$ & $\mathrm{Ca}^{2}$ & $\mathrm{Cs}^{2}$ & $\mathrm{~L}_{0}$ & $\mathrm{~L}_{1}$ & $\mathrm{~L}_{2}$ & $\lambda_{2}$ & $\pi_{\alpha}$ & Method \\
\hline & 0.5 & 5 & 0 & 1.600 & 1.111 & 0.8952 & 0.3253 & 0.3493 & $\mathrm{ME}$ \\
\hline & & & & 1.269 & 0.8553 & 0.8404 & 0.3000 & 0.2899 & SIM \\
\hline \multirow[t]{2}{*}{14} & 0.5 & 5 & 1 & 1.7507 & 1.2776 & 1.0493 & 0.3237 & 0.3526 & ME \\
\hline & & & & 1.4280 & 1.1590 & 1.0640 & 0.3463 & 0.3026 & SIM \\
\hline \multirow[t]{2}{*}{15} & 0.5 & 5 & 3 & 1.9570 & 1.5271 & 1.2600 & 0.3164 & 0.3671 & ME \\
\hline & & & & 1.9450 & 1.5920 & 1.2760 & 0.3254 & 0.3487 & SIM \\
\hline \multirow[t]{2}{*}{16} & 0.5 & 5 & 5 & 2.1163 & 1.7074 & 1.3837 & 0.3071 & 0.3859 & ME \\
\hline & & & & 2.3160 & 1.7810 & 1.2890 & 0.2965 & 0.4109 & SIM \\
\hline
\end{tabular}


Table 4 Hot Spot Routing

\begin{tabular}{|c|c|c|c|c|c|c|c|c|c|}
\hline \multirow[t]{2}{*}{$\begin{array}{c}\text { Banyan } \\
\text { No. }\end{array}$} & \multicolumn{3}{|c|}{$\begin{array}{c}\text { Input Data } \\
\left\{r_{i j}=0.02, i=0,1, \ldots, 7, j=1,2, \ldots 7\right\} \\
\left\{r_{i j}=0.86, i=0,1, \ldots, 7, j=0\right\} \\
N=8 ; \mu=1 ; K=9\end{array}$} & \multicolumn{6}{|c|}{ Output Statistics } \\
\hline & $\Lambda$ & $\mathrm{Ca}^{2}$ & $\mathrm{Cs}^{2}$ & $\mathrm{~L}_{0}$ & $\mathrm{~L}_{1}$ & $\mathrm{~L}_{2}$ & $\lambda_{2}$ & $\pi_{\alpha}$ & Method \\
\hline \multirow[t]{2}{*}{16} & 0.1 & 3 & 1 & 0.4705 & 0.9920 & 2.5333 & 0.6926 & 0.0078 & $\mathrm{ME}$ \\
\hline & & & & 0.4763 & 1.0240 & 2.6470 & 0.7062 & 0.0062 & SIM \\
\hline \multirow[t]{2}{*}{17} & 0.1 & 7 & 3 & 0.7625 & 1.6055 & 2.9622 & 0.6384 & 0.1076 & ME \\
\hline & & & & 0.7720 & 1.7230 & 3.0470 & 0.6431 & 0.1034 & SIM \\
\hline
\end{tabular}

Table 5 Warm Spot Routing

\begin{tabular}{|c|c|c|c|c|c|c|c|c|c|}
\hline $\begin{array}{c}\text { Banyan } \\
\text { No. }\end{array}$ & $\left\{\begin{array}{r}\left\{r_{i j}=0.1\right. \\
\left\{r_{i j}=\right.\end{array}\right.$ & $\begin{array}{l}\text { ut } D a \\
1, \ldots, 7 \\
=0,1 \text {. } \\
\iota=1 ;\end{array}$ & $\begin{array}{l}2, \ldots, 7\} \\
=0\}\end{array}$ & & & Output & Statistics & & \\
\hline \multirow{3}{*}{18} & $\Lambda$ & $\mathrm{Ca}^{2}$ & $\mathrm{Cs}^{2}$ & $\mathrm{~L}_{0}$ & $\mathrm{~L}_{1}$ & $\mathrm{~L}_{2}$ & $\lambda_{2}$ & $\pi_{\alpha}$ & Method \\
\hline & 0.3 & 7 & 3 & 1.9505 & 1.6403 & 1.8753 & 0.4523 & 0.1805 & ME \\
\hline & & & & 1.5670 & 1.4740 & 1.8440 & 0.7180 & 0.1432 & SIM \\
\hline \multirow[t]{2}{*}{19} & 0.5 & 5 & 5 & 3.6797 & 4.2865 & 4.5339 & 0.6926 & 0.2472 & $\mathrm{ME}$ \\
\hline & & & & 4.0050 & 4.4510 & 4.0750 & 0.6663 & 0.2733 & SIM \\
\hline
\end{tabular}

Table 6 First Stage QLDs

\begin{tabular}{c|cc|cc}
\hline \multicolumn{6}{c}{ Input Data: $\left\{\mathrm{Ca}^{2}=5, \mathrm{Cs}^{2}=3, \Lambda=0.5, \mu=1, K=5, N=8\right\} ;$} \\
\hline & \multicolumn{2}{|c}{ Aggregate $Q L D$} & \multicolumn{2}{c}{ Marginal $Q L D$} \\
\hline$n$ & $M E$ & SIM & ME & SIM \\
\hline 0 & 0.3770 & 0.3565 & 0.6351 & 0.5864 \\
1 & 0.1230 & 0.1412 & 0.1025 & 0.1417 \\
2 & 0.1190 & 0.1300 & 0.0849 & 0.1111 \\
3 & 0.1130 & 0.1189 & 0.0694 & 0.0793 \\
4 & 0.1070 & 0.1064 & 0.0558 & 0.0501 \\
5 & 0.1610 & 0.1470 & 0.0525 & 0.0313 \\
\hline
\end{tabular}


Table 7 Second Stage QLDs

\begin{tabular}{c|cc|cc}
\hline \multicolumn{5}{c}{ Input Data: $\left\{\mathrm{Ca}^{2}=5, \mathrm{Cs}^{2}=3, \Lambda=0.5, \mu=1, K=5, N=8\right\} ;$} \\
\hline & \multicolumn{2}{c}{ Aggregate $Q L D$} & \multicolumn{2}{c}{ Marginal $Q L D$} \\
\hline$n$ & $M E$ & SIM & ME & SIM \\
0 & 0.4220 & 0.3904 & 0.6589 & 0.6211 \\
1 & 0.1740 & 0.1859 & 0.1346 & 0.1605 \\
2 & 0.1360 & 0.1420 & 0.0887 & 0.1006 \\
3 & 0.1040 & 0.1082 & 0.0576 & 0.0609 \\
4 & 0.0790 & 0.0804 & 0.0364 & 0.0348 \\
5 & 0.0840 & 0.0931 & 0.0237 & 0.0222 \\
\hline
\end{tabular}

Table 8 Third Stage QLDs

\begin{tabular}{c|cc|cc}
\hline \multicolumn{5}{c}{ Input Data: $\left\{\mathrm{Ca}^{2}=5, \mathrm{Cs}^{2}=3, \Lambda=0.5, \mu=1, K=5, N=8\right\} ;$} \\
\hline & \multicolumn{2}{c}{ Aggregate $Q L D$} & \multicolumn{2}{c}{ Marginal $Q L D$} \\
\hline$n$ & ME & SIM & ME & SIM \\
0 & 0.4570 & 0.4572 & 0.6814 & 0.6734 \\
1 & 0.2000 & 0.2019 & 0.1479 & 0.1575 \\
2 & 0.1370 & 0.1368 & 0.0841 & 0.0858 \\
3 & 0.0920 & 0.0894 & 0.0471 & 0.0459 \\
4 & 0.0600 & 0.0583 & 0.0257 & 0.0236 \\
5 & 0.0530 & 0.0564 & 0.0138 & 0.0137 \\
\hline
\end{tabular}

Table 9 Buffer Assignment Biased for Stage 0

\begin{tabular}{|c|c|c|c|c|}
\hline \multicolumn{5}{|c|}{$\begin{array}{c}\text { Input Data: }\left\{\mathrm{Ca}^{2}=C s^{2}=5, \Lambda=0.1, \mu=1, N=8\right\} \\
\left\{r_{i j}=0.125, i, j=0,1, \ldots, 7\right\}\end{array}$} \\
\hline$K_{0}$ & $K_{1}$ & $K_{2}$ & $\begin{array}{l}\text { End-to-End } \\
\text { Delay }\end{array}$ & $C L P$ \\
\hline 9 & 9 & 9 & 9.0565 & 0.1170 \\
\hline 11 & 8 & 8 & 9.5461 & 0.0838 \\
\hline 13 & 7 & 7 & 9.9613 & 0.0616 \\
\hline 15 & 6 & 6 & 10.3868 & 0.0472 \\
\hline 17 & 5 & 5 & 10.9864 & 0.0392 \\
\hline 19 & 4 & 4 & 12.1656 & 0.0384 \\
\hline 21 & 3 & 3 & 15.3603 & 0.0541 \\
\hline
\end{tabular}


Table 10 Buffer Assignment Biased for Stage 1

\begin{tabular}{|c|c|c|c|c|}
\hline \multicolumn{5}{|c|}{$\begin{aligned} \text { Input Data: } & \left\{\mathrm{Ca}^{2}=C s^{2}=5, \Lambda=0.1, \mu=1, K=5, N=8\right\} ; \\
& \left\{r_{i j}=0.125, i, j=0,1, \ldots, 7\right\}\end{aligned}$} \\
\hline$K_{0}$ & $K_{1}$ & $K_{2}$ & $\begin{array}{c}\text { End-to-End } \\
\text { Delay }\end{array}$ & $C L P$ \\
\hline 9 & 9 & 9 & 9.0565 & 0.1170 \\
\hline 8 & 11 & 8 & 8.7827 & 0.1380 \\
\hline 7 & 13 & 7 & 8.4496 & 0.1648 \\
\hline 6 & 15 & 6 & 8.0488 & 0.1984 \\
\hline 5 & 17 & 5 & 7.5768 & 0.2401 \\
\hline 4 & 19 & 4 & 7.0342 & 0.2923 \\
\hline 3 & 21 & 3 & 6.4185 & 0.3585 \\
\hline
\end{tabular}

Table 11 Buffer Assignment Biased for Stage 2

\begin{tabular}{ccc|c|c}
\hline \multicolumn{5}{c}{ Input Data: $\left\{\mathrm{Ca}^{2}=C s^{2}=5, \Lambda=0.1, \mu=1, K=5, N=8\right\} ;$} \\
$\left\{r_{i j}=0.125, i, j=0,1, ., 7\right\}$
\end{tabular}

APPENDIX I DERIVATION OF AN ME QLD FOR A STABLE GER/GE/1 QUEUE

Consider a stable FCFS $\mathrm{GE}^{\mathrm{R}} / \mathrm{GE} / 1$ single server queue i depicted in Figure 4. The queue receives a multiple input of $\mathrm{R}$ streams with GE-type interarrival parameters.

$\left(\Lambda_{j i}, C a_{j i}^{2}\right), \quad j=1,2, \ldots, \mathrm{R}$. Moreover, the server provides GE-type service time with parameters $\left(\mu_{i}, C s_{i}^{2}\right)$. 
Table 12 Analytical Bounds over Simulation for $27 \times 27$ MINs

\begin{tabular}{|c|c|c|c|c|c|c|c|c|}
\hline \multirow[t]{2}{*}{$\begin{array}{l}\text { Banyan } \\
\text { No. }\end{array}$} & \multicolumn{3}{|c|}{$\begin{array}{c}\text { Input Data } \\
\left\{\begin{array}{c}\left.r_{i j}=0.037, i, j=0,1, \ldots, 26\right\} \\
N=27 ; \mu=1 ; K=9\end{array}\right.\end{array}$} & \multicolumn{5}{|c|}{ Output Statistics } \\
\hline & $\Lambda$ & $\mathrm{Ca}^{2}$ & $\mathrm{Cs}^{2}$ & $\mathrm{~L}_{0}$ & $\mathrm{~L}_{1}$ & $\mathrm{~L}_{2}$ & $\pi_{\alpha}$ & Method \\
\hline \multirow[t]{2}{*}{18} & 0.10 & 1 & 1 & 0.3330 & 0.3330 & 0.3330 & 0.0000 & ME \\
\hline & & & & 0.3339 & 0.3331 & 0.3333 & 0 & SIM \\
\hline \multirow[t]{2}{*}{19} & 0.25 & 1 & 1 & 0.9999 & 0.9999 & 0.9999 & 0.0001 & ME \\
\hline & & & & 0.9969 & 1.0330 & 0.9997 & 0.0001 & SIM \\
\hline \multirow[t]{2}{*}{20} & 0.10 & 7 & 1 & 0.8925 & 0.9937 & 0.8475 & 0.1241 & $\mathrm{ME}$ \\
\hline & & & & 0.9000 & 0.5600 & 0.4800 & 0.1234 & SIM \\
\hline \multirow[t]{2}{*}{21} & 0.25 & 7 & 1 & 1.9750 & 2.2800 & 1.8069 & 0.1973 & ME \\
\hline & & & & 2.2020 & 1.3600 & 1.2203 & 0.2022 & SIM \\
\hline \multirow[t]{2}{*}{22} & 0.10 & 3 & 7 & 0.7200 & 0.7021 & 0.6927 & 0.0112 & ME \\
\hline & & & & 0.7541 & 0.6918 & 0.6378 & 0.0126 & SIM \\
\hline \multirow[t]{2}{*}{23} & 0.25 & 7 & 3 & 2.1780 & 2.4270 & 1.9477 & 0.1921 & ME \\
\hline & & & & 2.3981 & 1.8912 & 1.5398 & 0.2091 & SIM \\
\hline
\end{tabular}

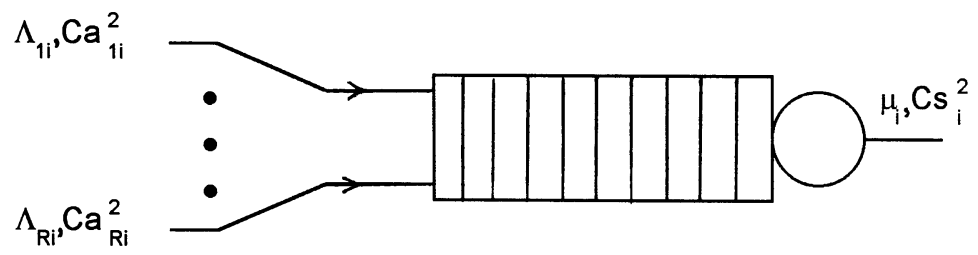

Figure 4. A stable $\left(\mathrm{GE}^{\mathrm{R}} / \mathrm{GE} / 1\right)$ Queue $\mathrm{i}$ 
Suppose all that is known about the $\mathrm{GE}^{\mathrm{R}} / \mathrm{GE} / 1$ queue is that the server utilisation, $\rho_{i}$, and MQL, $\widetilde{L}_{i}$. Entropy maximisation, subject to normalisation, utilisation and MQL constraints, implies that the $\mathrm{QLD}$ of the $\mathrm{GE}^{\mathrm{R}} / \mathrm{GE} / 1$ queue is given by

$p_{i}\left(n_{i}\right)=\frac{1}{Z_{i}} g_{i}^{h\left(n_{i}\right)} x_{i}^{n_{i}}$

where $Z_{i}=1 / p_{i}(0)$ is the normalising constant, $\mathrm{h}\left(\mathrm{n}_{\mathrm{i}}\right)$ is an auxiliary function defined by $h\left(n_{i}\right)=1$, if $\mathrm{n}_{i}>0$ or, 0 , otherwise, and $\left\{g_{i}, x_{i}\right\}$ are the Langriangian coefficients corresponding to utilisation and MQL constraints, respectively.

The server utilisation, $\rho_{i}$ is clearly expressed by

$\rho_{i}=\sum_{i=1}^{\mathrm{R}} \rho_{j i}, \quad \rho_{j i}=\Lambda_{j i} / \mu_{i}, \quad j=1,2, \ldots, \mathrm{R}$.

Moreover, an expression for the MQL, $\tilde{L}_{i}$, can be obtained from the generalised P-K expression for a stable $\mathrm{M}^{\mathrm{B}} / \mathrm{G} / 1$ queue [9], namely

$\widetilde{L}_{i}=\frac{\rho_{i}}{2}+\frac{1}{2\left(1-\rho_{i}\right)}\left(\rho_{i}^{2} C s_{i}^{2}+\rho_{i} b_{i}\left(C b_{i}^{2}+1\right)\right), i=1,2, \ldots, \mathrm{R}$,

where $b$ is the mean and $C b^{2}$ is the SCV of the bulk size distribution.

In the case of a number of arriving bulk Poisson streams with parameters $b_{j i}$ and $C b_{j i}^{2}, j=1,2, \ldots, \mathrm{R}$, respectively, the overall arrival stream is another bulk Poisson stream with mean, $b_{i}$, and SCV $C b_{i}^{2}$. The later parameters can be determined via the law of total moments, namely,

$b_{i}=\sum_{j=1}^{\mathrm{R}} b_{j i} p_{j i}, \quad i=1,2, \ldots, \mathrm{R}$,

where

$$
\begin{aligned}
& p_{j i}=\frac{\Lambda_{j i}}{\Lambda_{i}}, \Lambda_{i}=\sum_{j=1}^{\mathrm{R}} \Lambda_{j i}, \quad i=1,2, \ldots, \mathrm{R}, \\
& C b_{i}^{2}=\frac{b_{i}^{(2)}-b_{i}^{2}}{b_{i}^{2}}, \quad i=1,2, \ldots, \mathrm{R},
\end{aligned}
$$


where $b_{i}^{(2)}$ is the second moment of the bulk size of the overall stream, namely

$b_{i}^{(2)}=\sum_{j=l}^{R} b_{j i}^{(2)} p_{j i}, \quad i=1,2, \ldots, \mathrm{R}$,

where $b_{j i}^{(2)}$ is the second moment of the bulk size for steam $\mathrm{j}$.

Manipulations lead to relations

$\left(C b_{i}^{2}+1\right) \Lambda_{i} b_{i}=\sum_{j=1}^{R}\left(C b_{j i}^{2}+1\right) \Lambda_{j i} b_{j i}, \quad i=1,2, \ldots, \mathrm{R}$.

Substituting into the generalised P-K expression (A3) the following formula for the MQL of a stable $M^{B} / G$ / 1 queue with an aggregate of $\mathrm{R}$ multiple input streams is obtained:

$\widetilde{L}_{i}=\frac{\rho_{i}}{2}+\frac{1}{2\left(1-\rho_{i}\right)}\left(\rho_{i}^{2} C_{s i}^{2}+\sum_{j=1}^{R} \rho_{j i} b_{j i}\left(C b_{j i}^{2}+1\right)\right), \quad i=1,2, \ldots, \mathbf{R}$

Note that the superposition of R GE-type streams results into an overall bulk Poisson process, but the bulk size distribution is determined by a sum of geometrics. Moreover, the individual parameters of each bulk size distribution $b_{j i}$ and $C b_{j i}^{2}$ can be expressed by

$b_{j i}=\frac{C a_{j i}^{2}+1}{2}, i, j=1,2, \ldots, \mathrm{R}$,

and

$C b_{j i}^{2}=\frac{C a_{j i}^{2}-1}{C a_{j i}^{2}+1}, i, j=1,2, \ldots, \mathrm{R}$

Using expressions (A9) and (A10), formula (A8) becomes identical for MQL expression (4.9). Moreover, subtituting (A1) into the constriants of utilisation, $\rho_{i}$ and $\mathrm{MQL}, \widetilde{L}_{i}$, and carrying out some manipilations, Lagrangian coefficients $g_{i}$ and $x_{i}$ are determined via expressions (4.14). 


\section{APPENDIX II DERIVATION OF THE LARGRANGIAN COEFFICIENTS $\left\{y_{i}: i=1,2, \ldots, \mathrm{R}\right\}$ FOR THE CASE OF $\mathrm{R}=2$}

The Largrangian coefficients $\left\{y_{i}: i=1,2, \ldots, \mathrm{R}\right\}$ of the $\mathrm{S}_{\mathrm{RxR}}\left(\mathrm{GE}^{\mathrm{R}} / \mathrm{GE} / 1\right) / \mathrm{K}$ are determined by solving the set of nonlinear simutaneous equation (4.17). These can be written as

$$
C_{2}^{(i)}(\mathrm{K})[\mathbf{Y}]=C_{2}^{(i)}(\mathrm{K}), i=1,2, \ldots, \mathrm{R}
$$

where [Y] denotes the vector of $y_{i}{ }^{\prime} s$. In the case of $\mathbf{R}=2$, equation (A11) can be solved analytically as follows:

$$
\begin{aligned}
& C_{2}^{(1)}(\mathrm{K})[\mathbf{Y}]=g_{1} x_{1} y_{1}\left(x_{1}^{K-1}+x_{1}^{K-2} x_{2}+\ldots+x_{2}^{K-1}\right) \\
& +g_{2} x_{2} y_{2} g_{1} x_{1} y_{1}\left(x_{1}^{K-2}+x_{1}^{K-3} x_{2}+\ldots+x_{2}^{K-2}\right)
\end{aligned}
$$

and

$$
\begin{aligned}
& C_{2}^{(2)}(\mathrm{K})[\mathbf{Y}]=g_{2} x_{2} y_{2}\left(x_{1}^{K-1}+x_{1}^{K-2} x_{2}+\ldots+x_{2}^{K-1}\right) \\
& +g_{2} x_{2} y_{2} g_{1} x_{1} y_{1}\left(x_{1}^{K-2}+x_{1}^{K-3} x_{2}+\ldots+x_{2}^{K-2}\right)
\end{aligned}
$$

which leads to

$$
\begin{aligned}
& C_{2}^{(2)}(K)[\mathbf{Y}]-C_{2}^{(1)}(K)[\mathbf{Y}]=g_{2} x_{2} y_{2}\left(x_{1}^{K-1}+x_{1}^{K-2} x_{2}+\ldots+x_{2}^{K-1}\right) \\
& -g_{1} x_{1} y_{1}\left(x_{1}^{K-1}+x_{1}^{K-2} x_{2}+\ldots+x_{2}^{K-1}\right) .
\end{aligned}
$$

Equation (A.14) can be simplified by using the identitiy

$$
\left(x_{1}^{K-1}+x_{1}^{K-2} x_{2}+\ldots+x_{2}^{K-1}\right)\left(x_{1}-x_{2}\right)=\left(x_{1}^{K}-x_{2}^{K}\right) \text {. }
$$

To this end, solving (A.14) with respect to the Largrangian coeffcient $y_{2}$, it follows that (4.19) holds.

Substituting $y_{2}$ into (A.14), the following equation is obtained:

$$
\begin{aligned}
& 0=y_{1}^{2} g_{1}^{2} x_{1}^{2}\left(x_{1}^{K-1}-x_{2}^{K-1}\right) \\
& +y_{1}\left(\frac{g_{1} x_{1}\left(x_{1}^{K}-x_{2}^{K}\right)^{2}+g_{1} x_{1}\left(x_{1}^{K-1}-x_{2}^{K-1}\right)\left(C_{2}^{(2)}(\mathrm{K})-C_{2}^{(1)}(\mathrm{K})\right)}{\left(x_{1}^{K}-x_{2}^{K}\right)}\right)-C_{2}^{(1)}(\mathrm{K}) .
\end{aligned}
$$


Solving equation (A16) for $y_{1}$ and taking the positive root yields expression (4.18).

\section{APPENDIX III METHOD OF CALCULATING THE INPINS AND OUTPINS SETS}

Let Inpins $[i, m]$ be the set of external input pins of the Banyan Network which are connected to an interior input-port pin at position (i,m) of the array of input pins. Likewise let Inpins' $[i, \mathrm{~m}]$ be the set of external input pins that are linked to an interior output pin at position $(i, m)$ of the array of output pins. Let $S$ be the set of input pins that constitute the inputs of a particular switch. Each switch fully connects all its input-pins to its output pins, therefore the sets Inpins $[i, \mathrm{~m}]$ and Inpins $[\mathrm{i}, \mathrm{m}]$ are related as follows:

Inpins' $[i, m]=\bigcup_{k \in S} \operatorname{Inpins}[k, m]$.

Each interior input-port pin is connected to one output pin from the previous stage, which can be determined from the backwards topology matrix (BTM), i.e., input-pin at position (i,m) connects with output pin located at BTM [i,m], which is of course at stage (m-1).

Thus, each input pin 'inherits' its Inpin set from the output pin that it is connected to, i.e., Inpins $[\mathrm{i}, \mathrm{m}]=$ Inpins' $[\mathrm{BTM}[\mathrm{i}, \mathrm{m}], \mathrm{m}-1]$.

The input pins at the input edge of the network have only one element in their Inpins set, as they correspond to a particular external input pin, $i, i=0,1, \ldots, N-1$ i.e.,

Inpins $[\mathrm{i}, 0]=\{\mathrm{i}\}$

Thus Inpins sets at each level are obtained via the following procedure:

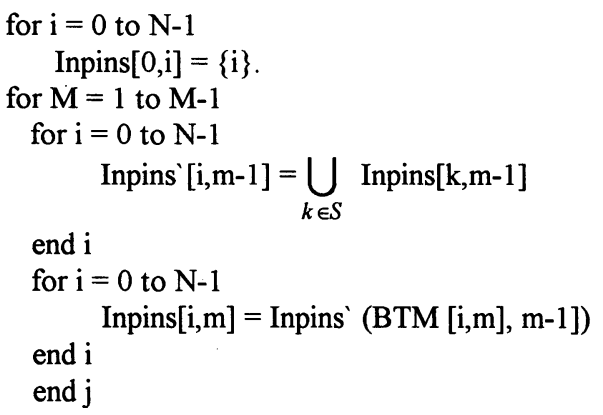

The method of calculating the Outpins sets is similar to the one for determining the Inpins sets but is applied in reverse order. 


\section{REFERENCES}

[1] Boyer P, Lehnert M R and Kuehn P J, "Queueing in an ATM basic switch element", Technical Report CNET-123-030-CD-CC, CNET, France, 1988.

[2] Eng K Y, Karol M K and Yeh Y-S, "A Growable Packet (ATM) Switch Architecture: Design Principles and Applications," IEEE Trans. Comm., 40, no. 2, pp. 423-430, Feb. 1992.

[3] Gelenbe, E. and Pujolle, G., The Behaviour of a single Queue in a General Queueing Network, Acta Informatica, 7, pp. 123-160, 1976.

[4] Harrison P G and Pinto A de C, "Blocking in Asynchronous, Buffered Banyan Networks," In Proc. of the IFIP WG 7.3 International Conference on the Performance of Distributed Systems and Integrated Communication Networks, Kyoto, Japan, ed. T Hasegawa, H Takagi and Y Takahashi, North-Holland, pp. 169-188, 1992.

[5] Harrison P G and Pinto A de C, "An Approximate Analysis of Asynchronous, Packetswitched Buffered Banyan Networks with Blocking," Performance Evaluation, 19, pp. 223-258, 1994.

[6] Hong S, Perros H G and Yamashita H, "A discrete-time queueing model of the shared buffer switch with bursty arrivals," Research Report, Computer Science Department, North Carolina State University, 1992.

[7] Jaynes, Prior probabilities, IEEE Trans. Syst. Sci. Cybern. SSC-4, pp.227-241, 1968.

[8] Karol M J and C-L I, "Performance Analysis of a Growable Architecture for Broad-Band Packet (ATM) Switch," IEEE Trans. Comm., 40, no. 2, pp. 431-439, Feb. 1992.

[9] Kleinrock, Queueing Systems, Vol.1: Theory, John Wiley and Sons, Inc., 1975.

[10] Kouvatsos, A Universal Maximum Entropy Algorithm for the Analysis of General Closed Networks, Computing Networking and Performance Evaluation, IFIP WG 7.3, T. Hasegawa, et al (Eds.), pp.113-124, North Holland, 1986.

[11] Kouvatsos, A Maximum Entropy Analysis of and the G/G/1 Queue at Equilibrium, J. Opl. Res. Soc., Vol.39, pp.183-200, 1988.

[12] Kouvatsos D and Xenios N, "MEM for Arbitrary Queueing Networks with Multiple General Servers and Repetitive-service Blocking," Performance Evaluation, 10, pp. 169-195. Sep. 1989.

[13] Kouvatsos and N. Tabet-Aouel, Product-Form Approximations for an Extended Class of General Closed Queueing Network, Performance '90, IFIP WG 7.3 and BCS, P. King et al (Eds.), North-Holland, pp.301-315, 1990. 
[14] Kouvatsos D and Denazis S G, "A Universal Building Block for the Approximate Analysis of a Shared Buffer ATM Switch Architecture," Annals of OR, 44, pp. 241-278, 1994.

[15] Kouvatsos, D.D., Tabet-Aouel, N. and Denazis, S.G., "ME-Based Approximations for General Discrete-Time Queueing Models", Performance Evaluation, Special Issue on Discrete-Time Models and Analysis Methods of Performance Evaluation, Vol.21, pp81109, 1994.

[16] Kouvatsos, Entropy Maximisation and Queueing Network Models, Annals of Operations Research, Special Issue on Queueing Networks, Vol. 48, pp.63-126, 1994.

[17] Kouvatsos, D.D. and Wilkinson, J., "A Product-Form Approximation for Discrete-Time Arbitrary Networks of ATM Switch Architectures",Performance Modelling and Evaluation of ATM Networks, IFIP Publication, Chapman and Hall, London, Vol.1, pp. 365-383, 1995.

[18] Kouvatsos, D.D. and Fretwell,R. "Closed Form Performance Distributions of a Discrete Time GI ${ }^{G} / \mathrm{D} / 1 / \mathrm{N}$ Queue with Correlated Traffic", Data Communications and their Performance, IFIP Publication, Fdida, S. and Onvural, R.O. (eds.), Chapman and Hall, London pp. 142-163, 1995.

[19] Kuwahara H, Endo N, Ogino M and Kozaki T, "A Shared Buffer Memory Switch for an ATM Exchange," In Proc. Int. Conf. on Communications, Boston, MA, pp. 441-444, June 1989.

[20] Lin, T., and Kleinrock L, "Performance Analysis of Finite-Buffered Multistage Interconnection Networks with a General Traffic Pattern," In Proc. ACM SIG-METRICS '91, pp. 68-89, 1991.

[21] Nojo and Watanabe H, A New Stage Method Getting Arbitrary Coefficient of Variation by two stages, Trans. IEICE 70, pp. 33-36, 1987.

[22] Shore, J.E., and Johnson, R.W., Axiomatic derivation of the principle of ME and the principle of minimum cross-entropy, IEEE Trans. Info. Theory IT-26, 1980.

[23] Sauer, Configuration of Computing Systems: An Approach Using Queueing Network Models, PhD Thesis, University of Texas, 1975.

[24] Szymanski T and Shaikh S, "Markov Chain Analysis of Packet-Switched Banyans with arbitrary Switch Sizes, Queue Sizes, Link Multiplicities and Speedups," 1989 IEEE INFOCOM, pp. 960-971, 1989.

[25] Tobagi F, "Fast Packet Switch Architectures for Broadband Integrated Services Digital Networks," Proc. of the IEEE, vol. 78, no. 1, Jan. 1990. 
[26] Yamashita H, Perros H G and Hong S, "Performance modelling of shared buffer ATM switch architecture," In Teletraffic and Datatraffic in a Period of Change, eds. Jensen and Iversen, North-Holland, 1991.

\section{BIOGRAPHIES}

Demetres Kouvatsos received the BSc degree in Mathematics from Athens National University in 1970, the MSc degree in Statistics from Victoria University of Manchester in 1971 and the $\mathrm{PhD}$ degree in Computation from UMIST, Institute of Science and Technology, University of Manchester in 1974. He is currently a Reader in Computer Systems Modelling, Department of Computing, University of Bradford. Since early 80 's, he pioneered new and cost-effective methodologies for the approximate analysis of arbitrary queueing network models of computer and communication systems. He has held a series of EPSRC (UK) and industrial research grants and is the author or co-author of many papers in the areas of queueing theory and systems performance modelling. He acted as the Chairman of the first four IFIP Workshops on "Performance Modelling and Evaluation of ATM Networks" (1993-96), and the Co-Chairman of the 3rd International Workshop in Queueing Networks with Finite Capacity" (1995).

Jeffrey Wilkinson received the BSc degree in Computer Science from Bradford University in 1992 and, subsequently joined the Computer Systems Performance Modelling Group, Bradford University, as $\mathrm{PhD}$ research student. Since January 1996 he is employed as a Research Assistant at the Department of Computing, University of Bradford, under an EPSRC (UK) funded project on the performance modelling of ATM networks. His research interests include queueing theory and performance modelling of computer and communication systems.

Peter Harrison is currently a Reader in Computing Science at Imperial College where he became a Lecturer in 1983. He graduated at Christ's College Cambridge as a Wrangler in Mathematics in 1972 and went on to gain Distinction in Part III of the Mathematical Tripos in 1973, winning the Mayhew prize. He obtained his $\mathrm{PhD}$ in Computing Science at Imperial College in 1979. He has researched into analytical performance modelling techniques and algebraic program transformation for some fifteen years, visiting IBM Research Centers for two summers in the last decade. He has written two books, had over 70 research papers published in the areas of performance modelling and functional programming and held a series of research grants.

Madhu Bhabuta received her MEng degree from Imperial College in 1994. She was awarded a scholarship from the ESPRIT Basic Research group in November 1994 to fund work leading to a $\mathrm{PhD}$ degree. She is currently a Research Assistant at Imperial College and is supported by EPSRC. Her areas of interest include performance modelling, ATM traffic modelling and stochastic process algebras. 